\title{
Search for doubly heavy dibaryons in the quark delocalization color screening model
}

\author{
Zhuocheng Xia $\odot,{ }^{1, *}$ Saijun Fan, ${ }^{1, \dagger}$ Xinmei Zhu, ${ }^{2, \ddagger}$ Hongxia Huang $\odot,{ }^{1, \S}$ and Jialun Ping ${ }^{1, \|}$ \\ ${ }^{1}$ Department of Physics, and Jiangsu Key Laboratory for Numerical Simulation of Large Scale Complex Systems, \\ Nanjing Normal University, Nanjing 210023, People's Republic of China \\ ${ }^{2}$ Department of Physics, Yangzhou University, Yangzhou 225009, People's Republic of China
}

(Received 4 June 2021; revised 27 December 2021; accepted 25 January 2022; published 3 February 2022)

\begin{abstract}
We perform a systemical investigation of the low-lying doubly heavy dibaryon systems with strange $S=$ 0 , isospin $I=0,1,2$, and the angular momentum $J=0,1,2,3$ in the quark delocalization color screening model. We find the effect of channel-coupling cannot be neglected in the study of the multi-quark systems. Several bound systems are obtained. They are the doubly charm dibaryon systems with $I J=00, I J=01$, and $I J=02$, and the corresponding energies are 4590, 4720, and $4764 \mathrm{MeV}$, respectively; and the doubly bottom dibaryon systems with $I J=00, I J=02$, and $I J=13$, and the corresponding energies are 11219,11416 , and $11633 \mathrm{MeV}$, respectively. Besides, six resonance states are obtained, which are $I J=00 N \Xi_{c c}$ and $N \Xi_{b b}$ with resonance masses of 4730 and $11411 \mathrm{MeV}$ respectively, $I J=11 N \Xi_{c c}^{*}$ and $N \Xi_{b b}^{*}$ with resonance masses of 4775 and $11432 \mathrm{MeV}$ respectively, and $I J=12 \Sigma_{c} \Sigma_{c}^{*}$ and $\Sigma_{b} \Sigma_{b}^{*}$ with resonance masses of 4935 and $11626 \mathrm{MeV}$ respectively. All these heavy dibaryons are worth searching for in experiments, although it will be a challenging work.
\end{abstract}

DOI: 10.1103/PhysRevC.105.025201

\section{INTRODUCTION}

A worldwide theoretical and experimental effort to search for dibaryons has lasted a long time. Although the research on the dibaryon has experienced several ups and downs in its history, dibaryons have received renewed interest in recent years. The well-known dibaryon resonance $d^{*}$ was repeatedly observed by the Wide Angle Shower Apparatus (WASA) detector at the Cooler Synchrotron (COSY) [1-5], and extensively investigated within various theoretical approaches [6-13]. Another dibaryon $N \Omega$ was proposed as a narrow resonance in a relativistic quark model [14] and was investigated by other quark models [15-20], as well as the lattice QCD $[21,22]$. The progress of the $N \Omega$ searches by the STAR experiment at the Relativistic Heavy-Ion Collider (RHIC) favored the existence of $N \Omega$ [23]. Besides, the study of the strong interaction among hadrons at the Large Hadron Collider (LHC) by the ALICE Collaboration also supported the possibility of forming the $N \Omega$ state [24].

In the past decade, many near-threshold charmonium-like states called $X Y Z$ particles have been observed, triggering lots

\footnotetext{
*xiazach003@outlook.com

†69985589@qq.com

${ }^{\ddagger}$ zxm_yz@126.com

${ }^{\S}$ Corresponding author: hxhuang@njnu.edu.cn

"Corresponding author: jlping@ @jnu.edu.cn
}

Published by the American Physical Society under the terms of the Creative Commons Attribution 4.0 International license. Further distribution of this work must maintain attribution to the author $(s)$ and the published article's title, journal citation, and DOI. Funded by $S C O A P^{3}$. of studies on the molecule-like bound states containing heavy quark hadrons. Such studies will give further information on the hadron-hadron interactions. In the heavy-quark sector, the large masses of the heavy quarks reduce the kinetic energy of the system, which makes them easier to form bound states. Therefore, extending the dibaryon research to the heavy quark sector is spontaneous.

On the hadron level, the dibaryon systems with one heavy quark such as $N \Sigma_{c}, N \Lambda_{c}$, and so on, have been investigated in the realistic phenomenological nucleon-nucleon interaction model [25] and the one-boson-exchange model [26]. The dibaryon systems with two heavy quarks were also researched in the one-pion-exchange model [27] and oneboson-exchange model $[28,29]$. The possibility of existing deuteron-like dibaryons with heavy quarks, such as $N \Xi_{c c}$, $\Xi \Xi_{c c}$, and so on, were investigated by several realistic phenomenological nucleon-nucleon interaction models [25,30]. In Refs. [31,32], the deuteron-like states composed of two doubly charmed baryons $\boldsymbol{\Xi}_{c c} \boldsymbol{\Xi}_{c c}$ and $\boldsymbol{\Xi}_{c c} \bar{\Xi}_{c c}$ were systematically studied within the one-boson-exchange model.

On the quark level, the $N \Lambda_{c}$ system and the $H$-like dibaryon state $\Lambda_{c} \Lambda_{c}$ have been studied in the quark delocalization color screening model [33,34]. References [35-37] have also studied these dibaryons within the chiral constituent quark model. In Refs. [38,39], the two- and three-baryon systems with heavy quarks were investigated by using the baryon-baryon interaction derived from the constituent quark model. Besides, the possible $N \Omega$-like dibaryons $N \Omega_{c c c}$ and $N \Omega_{b b b}$ were investigated within both the chiral quark model and quark delocalization color screening model [40]. Recently, Junnarkar and Mathur reported the first lattice QCD study of deuteron-like dibaryons with heavy quark flavors [41], and suggested that the dibaryons $\Omega_{c} \Omega_{c c}(\operatorname{sscscc})$, 
$\Omega_{b} \Omega_{b b}(s s b s b b)$, and $\Omega_{c c b} \Omega_{c b b}(c c b c b b)$ were stable under strong and electromagnetic interactions. They also found that the binding of these dibaryons became stronger as they became heavier in mass. However, the distinct conclusion was claimed in the work of Ref. [42], where the authors explored the possibility of very heavy dibaryons with three charm quarks and three beauty quarks (bbbccc) in potential models, and concluded that there was no evidence for any stable state in such very heavy flavored six-quark system. Additionally, the existence of fully heavy dibaryons were also investigated in the constituent quark model [43].

Quantum chromodynamics (QCD) is widely accepted as a fundamental theory to study strong interaction. However, for hadron-hadron interactions and exotic quark states, it is difficult to use QCD directly to study low-energy hadronic interaction because of the nonperturbative complication. Therefore, it has triggered kinds of QCD-inspired models, which study the muliquark systems from the physical perspective. The quark delocalization color screening model (QDCSM) is one of the representations of the constituent quark models, which was developed in the 1990s, aiming to explain the similarities between nuclear and molecular force [44]. The model modifies the Hamiltonian by introducing the shielding effect of color screen and expands Hilbert space by considering the quark delocalization between two quark clusters. This model has been well applied to describe the properties of the deuteron, study the $N N$ and $Y N$ interactions, and investigate the dibaryon candidates [10]. It has also been extended to observe the dibaryon states with heavy quarks, such as the $N \Lambda_{c}$ and $N \Lambda_{b}$ systems [33], and the possible $H$-like dibaryon states $\Lambda_{c} \Lambda_{c}$ and $\Lambda_{b} \Lambda_{b}$ [34], the $N \Omega$-like dibaryons $N \Omega_{c c c}$ and $N \Omega_{b b b}$ [40], the fully heavy dibaryons [43], and so on. It is interesting to search for more dibaryons in heavy quark sector within the frame of QDCSM.

In this work, we further study the doubly heavy dibaryons systematically in the QDCSM. Extension of the study to the bottom case is also interesting and is performed too. Through our calculation, we can look for possible doubly heavy dibaryons, which will provide more information for the experimental work. The structure of this paper is as follows. After the introduction, we have a simple description of the QDCSM in Sec. II. Section III is the numerical results and discussions. The summery is given in the last section.

\section{MODEL HAMILTONIAN AND WAVE FUNCTION}

\section{A. Quark delocalization color screening model}

The quark delocalization and color screening model (QDCSM) has been described in detail in Ref. [44]. Here,we just present the Hamiltonian of the model:

$$
\begin{aligned}
& H=\sum_{i=1}^{6}\left(m_{i}+\frac{p_{i}^{2}}{2 m_{i}}\right)-T_{c}+\sum_{i<j}\left[V^{G}\left(r_{i j}\right)+V^{\chi}\left(r_{i j}\right)+V^{C}\left(r_{i j}\right)\right] \\
& V^{G}\left(r_{i j}\right)=\frac{1}{4} \alpha_{s} \lambda_{i} \cdot \lambda_{j}\left[\frac{1}{r_{i j}}-\frac{\pi}{2}\left(\frac{1}{m_{i}^{2}}+\frac{1}{m_{j}^{2}}+\frac{4 \sigma_{i} \cdot \sigma_{j}}{3 m_{i} m_{j}}\right) \delta\left(r_{i j}\right)-\frac{3}{4 m_{i} m_{j} r_{i j}^{3}} S_{i j}\right], \\
& V^{\mathrm{OBE}}\left(r_{i j}\right)=v^{\pi}\left(r_{i j}\right) \sum_{a=1}^{3} \lambda_{i}^{a} \cdot \lambda_{j}^{a}+v^{K}\left(r_{i j}\right) \sum_{a=4}^{7} \lambda_{i}^{a} \cdot \lambda_{j}^{a}+v^{\eta}\left(r_{i j}\right)\left[\left(\lambda_{i}^{8} \cdot \lambda_{j}^{8}\right) \cos \theta_{P}-\left(\lambda_{i}^{0} \cdot \lambda_{j}^{0}\right) \sin \theta_{P}\right] \\
& v^{\chi}\left(r_{i j}\right)=\frac{1}{3} \alpha_{c h} \frac{\Lambda^{2}}{\Lambda^{2}-m_{\chi}^{2}} m_{\chi}\left\{\left[Y\left(m_{\chi} r_{i j}\right)-\frac{\Lambda^{3}}{m_{\chi}^{3}} Y\left(\Lambda r_{i j}\right)\right] \sigma_{i} \cdot \sigma_{j}+\left[H\left(m_{\chi} r_{i j}\right)-\frac{\Lambda^{3}}{m_{\chi}^{3}} H\left(\Lambda r_{i j}\right)\right] S_{i j}\right\} \mathbf{F}_{i} \cdot \mathbf{F}_{j}, \quad \chi=\pi, K, \eta \\
& V^{C}\left(r_{i j}\right)=-a_{c} \lambda_{i} \cdot \lambda_{j}\left[f\left(r_{i j}\right)+V_{0}\right], \\
& f\left(r_{i j}\right)= \begin{cases}r_{i j}^{2} & \text { if } i, j \text { occur in the same baryon orbit } \\
\frac{1-e^{-\mu_{i j} r_{i j}^{2}}}{\mu_{i j}} & \text { if } i, j \text { occur in different baryon orbits }\end{cases} \\
& S_{i j}=\frac{\left(\boldsymbol{\sigma}_{i} \cdot \mathbf{r}_{i j}\right)\left(\boldsymbol{\sigma}_{j} \cdot \mathbf{r}_{i j}\right)}{r_{i j}^{2}}-\frac{1}{3} \boldsymbol{\sigma}_{i} \cdot \boldsymbol{\sigma}_{j},
\end{aligned}
$$

where $S_{i j}$ is the quark tensor operator. We only consider the $S$-wave systems at present, so the tensor force dose not work here. $Y(\chi)$ and $H(\chi)$ are standard Yukawa functions [45]; $T_{C M}$ is the kinetic energy of the center; and $\alpha_{s}$ is the quarkgluon coupling constant. In order to cover the wide energy range from light, strange to heavy quarks, one introduces an effective scale-dependent quark-gluon coupling constant $\alpha_{s}(\mu)[46]:$

$$
\alpha_{s}(\mu)=\frac{\alpha_{0}}{\ln \left(\frac{\mu^{2}+\mu_{0}^{2}}{\Lambda_{0}^{2}}\right)},
$$

where $\mu$ is the reduced mass of the interacting quark pair. The coupling constant $g_{\text {ch }}$ for scalar chiral field is determined from the $N N \pi$ coupling constant through

$$
\frac{g_{\mathrm{ch}}^{2}}{4 \pi}=\left(\frac{3}{5}\right)^{2} \frac{g_{\pi N N}^{2}}{4 \pi} \frac{m_{u, d}^{2}}{m_{N}^{2}}
$$

All other symbols have their usual meanings in the above expressions. All parameters are from our previous study of the $H$-like dibaryon state $\Lambda_{c} \Lambda_{c}$ [34], and they are listed in Table I. Here, in addition to Goldstone bosons of the hidden approximate chiral symmetry of QCD, the additional $D$ meson can exchange between $u / d$ and $c$ quarks, $D_{s}$ meson can exchange between $s$ and $c$ quarks, and the $\eta_{c}$ meson can exchange between two of the $u, d, s$, and $c$ quarks. In order to incorporate the charm quark and study the effect of the $D, D_{s}$, 
TABLE I. Model parameters $m_{\pi}=0.7 \mathrm{fm}^{-1}, m_{K}=2.51 \mathrm{fm}^{-1}, m_{\eta}=2.77 \mathrm{fm}^{-1}, \Lambda_{\pi}=4.2 \mathrm{fm}^{-1}, \Lambda_{K}=5.2 \mathrm{fm}^{-1}, \Lambda_{\eta}=5.2 ; \mathrm{fm}^{-1}$, $\alpha_{\mathrm{ch}}=0.027$.

\begin{tabular}{|c|c|c|c|c|c|c|c|c|c|c|}
\hline & $\begin{array}{c}b \\
(\mathrm{fm})\end{array}$ & $\begin{array}{c}m_{u, d} \\
(\mathrm{MeV})\end{array}$ & $\begin{array}{c}m_{s} \\
(\mathrm{MeV})\end{array}$ & $\begin{array}{c}m_{c} \\
(\mathrm{MeV})\end{array}$ & $\begin{array}{c}m_{b} \\
(\mathrm{MeV})\end{array}$ & $\begin{array}{c}a_{c} \\
\left(\mathrm{MeV} \mathrm{fm}^{-2}\right)\end{array}$ & $\begin{array}{c}V_{0} \\
\left(\mathrm{fm}^{2}\right)\end{array}$ & $\begin{array}{c}\Lambda_{0} \\
\left(\mathrm{fm}^{-1}\right)\end{array}$ & $\begin{array}{c}u_{0} \\
(\mathrm{MeV})\end{array}$ & $\begin{array}{c}\alpha_{0} \\
(\mathrm{MeV})\end{array}$ \\
\hline QDCSM & 0.6 & 313 & 539 & 1732 & 5070 & 18.5283 & -0.3333 & 1.7225 & 445.8512 & 0.7089 \\
\hline
\end{tabular}

and $\eta_{c}$ mesons exchange interactions, we extend the model from $S U(3)$ to $S U(4)$, and add the interaction of these heavy mesons interactions as follows:

$$
\begin{aligned}
V^{\mathrm{HM}}\left(r_{i j}\right)= & v^{D}\left(r_{i j}\right) \sum_{a=9}^{12} \lambda_{i}^{a} \cdot \lambda_{j}^{a}+v^{D_{s}}\left(r_{i j}\right) \sum_{a=13}^{14} \lambda_{i}^{a} \cdot \lambda_{j}^{a} \\
& +v^{\eta_{c}}\left(r_{i j}\right) \lambda_{i}^{15} \cdot \lambda_{j}^{15}
\end{aligned}
$$

where the formulas of the $v^{D}\left(r_{i j}\right), v^{D_{s}}\left(r_{i j}\right)$, and $v^{\eta_{c}}\left(r_{i j}\right)$ are the the same as the Eq. (4), in which the $\chi=D, D_{s}, \eta_{c}$ respectively. The extension is made in the spirit of the phenomenological approach of Refs. [47,48]. Here, we should emphasize that neither the $K$ meson nor the $D_{s}$ meson exchange contributes to the doubly heavy dibaryon systems in the present work, because we only consider the dibaryons composed of four nonstrange quarks and two heavy quarks. More doubly heavy dibaryon systems which contain strange quarks will be studied in future work. Besides, we do not readjust the model parameters after adding the heavy mesons exchange interactions. The binding energy is obtained by $B=$ $E-E_{\text {th }}$, where $E$ and $E_{\text {th }}$ are the energy and the threshold of the state respectively, so the mass difference between the theoretical and the experimental values will be eliminated by doing this subtraction. The masses of the charmed baryons without and with the heavy mesons exchange interactions are listed in Table II. In addition, the $D, D_{s}$, and $\eta_{c}$ mesons exchange do not work in the bottomed baryons, so the masses of the bottomed baryons listed in Table II do not include these heavy meson exchange.

\section{B. Wave function}

The resonating group method (RGM) [50,51] and generating coordinates method [52] are used to carry out a dynamical calculation. The main feature of the RGM for two-cluster systems is that it assumes that two clusters are frozen inside and only considers the relative motion between the two clusters, so the conventional ansatz for the two-cluster wave functions is

$$
\psi_{6 q}=\mathcal{A}\left[\left[\phi_{B_{1}} \phi_{B_{2}}\right]^{[\sigma] I S} \otimes \chi_{L}(\boldsymbol{R})\right]^{J}
$$

where the symbol $\mathcal{A}$ is the antisymmetrization operator. With the $S U(4)$ extension, both the light and heavy quarks are considered as identical particles. So $\mathcal{A}=1-9 P_{36}$. $[\sigma]=[222]$ gives the total color symmetry and all other symbols have their usual meanings. $\phi_{B_{i}}$ is the three-quark cluster wave function. From the variational principle, after variation with respect to the relative motion wave function $\chi(\mathbf{R})=\sum_{L} \chi_{L}(\mathbf{R})$, one obtains the RGM equation

$$
\int H\left(\mathbf{R}, \mathbf{R}^{\prime}\right) \chi\left(\mathbf{R}^{\prime}\right) d \mathbf{R}^{\prime}=E \int N\left(\mathbf{R}, \mathbf{R}^{\prime}\right) \chi\left(\mathbf{R}^{\prime}\right) d \mathbf{R}^{\prime},
$$

where $H\left(\mathbf{R}, \mathbf{R}^{\prime}\right)$ and $N\left(\mathbf{R}, \mathbf{R}^{\prime}\right)$ are Hamiltonian and norm kernels. By solving the RGM equation, we can get the energies $E$ and the wave functions. In fact, it is not convenient to work with the RGM expressions. Then, we expand the relative motion wave function $\chi(\mathbf{R})$ by using a set of Gaussians with different centers,

$$
\begin{aligned}
\chi_{L}(\boldsymbol{R})= & \frac{1}{\sqrt{4 \pi}}\left(\frac{3}{2 \pi b^{2}}\right)^{3 / 4} \sum_{i=1}^{n} C_{i} \\
& \times \int \exp \left[-\frac{3}{4 b^{2}}\left(\boldsymbol{R}-\boldsymbol{S}_{i}\right)^{2}\right] Y_{L M}\left(\hat{\boldsymbol{S}}_{i}\right) d \hat{\boldsymbol{S}}_{i},
\end{aligned}
$$

where $L$ is the orbital angular momentum between two clusters, and $\boldsymbol{S}_{i}, i=1,2, \ldots, n$ are the generator coordinates, which are introduced to expand the relative motion wave function. By including the center of mass motion,

$$
\phi_{C}\left(\boldsymbol{R}_{C}\right)=\left(\frac{6}{\pi b^{2}}\right)^{3 / 4} e^{-\frac{3 R_{C}^{2}}{b^{2}}}
$$

the ansatz Eq. (11) can be rewritten as

$$
\begin{aligned}
\psi_{6 q}= & \mathcal{A} \sum_{i=1}^{n} C_{i} \int \frac{d \hat{\boldsymbol{S}}_{i}}{\sqrt{4 \pi}} \prod_{\alpha=1}^{3} \phi_{\alpha}\left(\boldsymbol{S}_{i}\right) \prod_{\beta=4}^{6} \phi_{\beta}\left(-\boldsymbol{S}_{i}\right) \\
& \times\left[\left[\chi_{I_{1} S_{1}}\left(B_{1}\right) \chi_{I_{2} S_{2}}\left(B_{2}\right)\right]^{I S} Y_{L M}\left(\hat{\boldsymbol{S}}_{i}\right)\right]^{J} \\
& \times\left[\chi_{c}\left(B_{1}\right) \chi_{c}\left(B_{2}\right)\right]^{[\sigma]},
\end{aligned}
$$

TABLE II. The masses (in MeV) of the baryons without/with the heavy mesons exchange interactions. Experimental values are taken from the Particle Data Group (PDG) [49].

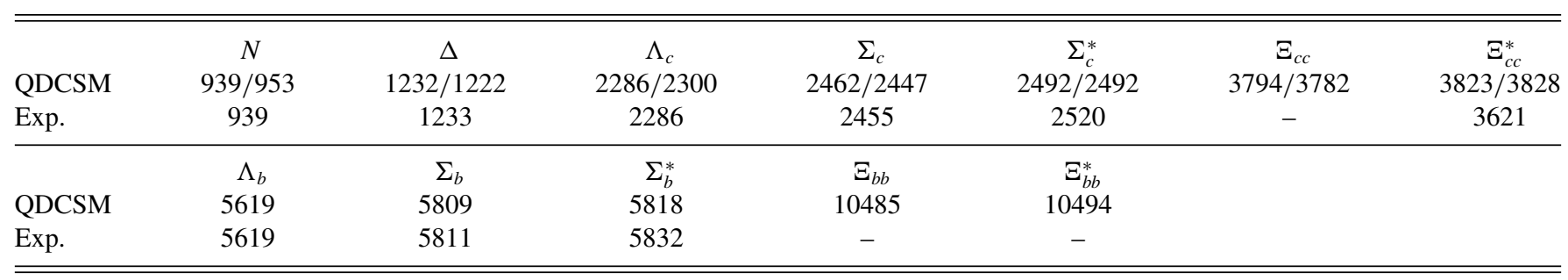


where $\chi_{I_{1} S_{1}}$ and $\chi_{I_{2} S_{2}}$ are the product of the flavor and spin wave functions, and $\chi_{c}$ is the color wave function. These will be shown in detail later. $\phi_{\alpha}\left(\boldsymbol{S}_{i}\right)$ and $\phi_{\beta}\left(-\boldsymbol{S}_{i}\right)$ are the single-particle orbital wave functions with different reference centers:

$$
\begin{gathered}
\phi_{\alpha}\left(\boldsymbol{S}_{i}\right)=\left(\frac{1}{\pi b^{2}}\right)^{\frac{3}{4}} e^{-\frac{\left(r_{\alpha}-S_{i} / 2\right)^{2}}{2 b^{2}}}, \\
\phi_{\beta}\left(-\boldsymbol{S}_{i}\right)=\left(\frac{1}{\pi b^{2}}\right)^{\frac{3}{4}} e^{-\frac{\left(r_{\beta}+S_{i} / 2\right)^{2}}{2 b^{2}}} .
\end{gathered}
$$

With the reformulated ansatz, Eq. (15), the RGM equation (12) becomes an algebraic eigenvalue equation:

$$
\sum_{j} C_{j} H_{i, j}=E \sum_{j} C_{j} N_{i, j},
$$

where $H_{i, j}$ and $N_{i, j}$ are the Hamiltonian matrix elements and overlaps, respectively. By solving the generalized eigenproblem, we can obtain the energy and the corresponding wave functions of the dibaryon system.

The quark delocalization in QDCSM is realized by specifying the single-particle orbital wave function of QDCSM as a linear combination of left and right Gaussians, and the single-particle orbital wave functions used in the ordinary quark cluster model,

$$
\begin{aligned}
\psi_{\alpha}\left(\boldsymbol{S}_{i}, \epsilon\right) & =\left[\phi_{\alpha}\left(\boldsymbol{S}_{i}\right)+\epsilon \phi_{\alpha}\left(-\boldsymbol{S}_{i}\right)\right] / N(\epsilon), \\
\psi_{\beta}\left(-\boldsymbol{S}_{i}, \epsilon\right) & =\left[\phi_{\beta}\left(-\boldsymbol{S}_{i}\right)+\epsilon \phi_{\beta}\left(\boldsymbol{S}_{i}\right)\right] / N(\epsilon), \\
N(\epsilon) & =\sqrt{1+\epsilon^{2}+2 \epsilon e^{-S_{i}^{2} / 4 b^{2}}} .
\end{aligned}
$$

Here the delocalization parameter $\epsilon\left(S_{i}\right)$ is determined by the dynamics of the multiquark system. In this way, the system can choose its most favorable configuration through its own dynamics in a larger Hilbert space. It has been used to explain the crossover transition between hadron phase and quarkgluon plasma phase [53].

The spin wave functions of a $q^{3}$ cluster is labeled as $\chi_{s, s_{z}}^{\sigma}$, where $s$ and $s_{z}$ are the spin quantum number and the third component, respectively:

$$
\begin{aligned}
\chi_{\frac{3}{2}, \frac{3}{2}}^{\sigma} & =\alpha \alpha \alpha, \\
\chi_{\frac{3}{2}, \frac{1}{2}}^{\sigma} & =\frac{1}{\sqrt{3}}(\alpha \alpha \beta+\alpha \beta \alpha+\beta \alpha \alpha), \\
\chi_{\frac{3}{2},-\frac{1}{2}}^{\sigma} & =\frac{1}{\sqrt{3}}(\alpha \beta \beta+\beta \alpha \beta+\beta \beta \alpha), \\
\chi_{\frac{3}{2},-\frac{3}{2}}^{\sigma} & =\beta \beta \beta, \\
\chi_{\frac{1}{2}, \frac{1}{2}}^{\sigma 1} & =\sqrt{\frac{1}{6}}(2 \alpha \alpha \beta-\alpha \beta \alpha-\beta \alpha \alpha), \\
\chi_{\frac{1}{2}, \frac{1}{2}}^{\sigma 2} & =\sqrt{\frac{1}{2}}(\alpha \beta \alpha-\beta \alpha \alpha), \\
\chi_{\frac{1}{2},-\frac{1}{2}}^{\sigma 1} & =\sqrt{\frac{1}{6}}(\alpha \beta \beta+\beta \alpha \beta-2 \beta \beta \alpha), \\
\chi_{\frac{1}{2},-\frac{1}{2}}^{\sigma 2} & =\sqrt{\frac{1}{2}}(\alpha \beta \beta-\beta \alpha \beta) .
\end{aligned}
$$

The flavor wave functions of the $q^{3}$ cluster $\chi_{I, I_{z}}^{f}$ ( $I$ and $I_{z}$ are the isospin quantum number and the third component, respectively) are as follows. Here, both the light and heavy quarks are considered as identical particles with the $S U(4)$ extension:

$$
\begin{aligned}
& \chi_{\frac{1}{2}, \frac{1}{2}}^{f 1}=\sqrt{\frac{1}{6}}(2 u u d-u d u-d u u), \\
& \chi_{\frac{1}{2}, \frac{1}{2}}^{f 2}=\sqrt{\frac{1}{2}}(u d u-d u u), \\
& \chi_{\frac{1}{2},-\frac{1}{2}}^{f 1}=\sqrt{\frac{1}{6}}(u d d+d u d-2 d d u), \\
& \chi_{\frac{1}{2},-\frac{1}{2}}^{f 2}=\sqrt{\frac{1}{2}}(u d d-d u d), \\
& \chi_{\frac{3}{2}, \frac{3}{2}}^{f}=u u u, \\
& \chi_{\frac{3}{2}, \frac{1}{2}}^{f}=\sqrt{\frac{1}{3}}(u u d+u d u+d u u), \\
& \chi_{\frac{3}{2},-\frac{1}{2}}^{f}=\sqrt{\frac{1}{3}}(u d d+d u d+d d u), \\
& \chi_{\frac{3}{2},-\frac{3}{2}}^{f}=d d d, \\
& \chi_{0,0}^{f 1}=\frac{1}{2}(u c d+c u d-c d u-d c u), \\
& \chi_{0,0}^{f 2}=\sqrt{\frac{1}{12}}(2 u d c-2 d u c+c d u+u c d-c u d-d c u), \\
& \chi_{1,1}^{f 1}=\sqrt{\frac{1}{6}}(2 u u c-u c u-c u u), \\
& \chi_{1,1}^{f 2}=\sqrt{\frac{1}{2}}(u c u-c u u), \\
& \chi_{1,0}^{f 1}=\sqrt{\frac{1}{12}}(2 u d c+2 d u c-c d u-u c d-c u d-d c u), \\
& \chi_{1,0}^{f 2}=\frac{1}{2}(u c d+d c u-c d u-c u d), \\
& \chi_{1,-1}^{f 1}=\sqrt{\frac{1}{6}}(2 d d c-d s d-c d d), \\
& \chi_{1,-1}^{f 2}=\sqrt{\frac{1}{2}}(d c d-c d d) \\
& \chi_{1,1}^{f 3}=\sqrt{\frac{1}{3}}(u u c+u c u+c u u), \\
& \chi_{1,0}^{f 3}=\sqrt{\frac{1}{6}}(u d c+d u c+c d u+u c d+c u d+d c u), \\
& \chi_{1,-1}^{f 3}=\sqrt{\frac{1}{3}}(d d c+d c d+c d d), \\
& \chi_{\frac{1}{2}, \frac{1}{2}}^{f 3}=\sqrt{\frac{1}{6}}(u c c+c u c-2 c c u), \\
& \chi_{\frac{1}{2}, \frac{1}{2}}^{f 4}=\sqrt{\frac{1}{2}}(u c c-c u c),
\end{aligned}
$$




$$
\begin{aligned}
\chi_{\frac{1}{2},-\frac{1}{2}}^{f 3} & =\sqrt{\frac{1}{6}}(d c c+c d c-2 c c d), \\
\chi_{\frac{1}{2},-\frac{1}{2}}^{f 4} & =\sqrt{\frac{1}{2}}(d c c-c d c), \\
\chi_{\frac{1}{2}, \frac{1}{2}}^{f 5} & =\sqrt{\frac{1}{3}}(u c c+c u c+c c u), \\
\chi_{\frac{1}{2},-\frac{1}{2}}^{f 5} & =\sqrt{\frac{1}{3}}(d c c+c d c+c c d) .
\end{aligned}
$$

The color wave function of a color-singlet $q^{3}$ cluster is

$$
\chi^{c}=\sqrt{\frac{1}{6}}(r g b-r b g+g b r-g r b+b r g-b g r) .
$$

The total flavor-spin-color wave function of the dibaryon system can be acquired by substituting the wave functions of the flavor, the spin, and the color parts according to the given quantum number of the system. Here, we can take the $I J=$ 00 system as an example to show the construction of wave function. There are four channels for the $I J=00$ system, and the total flavor-spin-color wave function for each channel is shown as follows:

$$
\begin{aligned}
& \left|\Sigma_{c} \Sigma_{c}\right\rangle=\sqrt{\frac{1}{6}}\left[\phi_{1, \frac{1}{2}}^{\Sigma_{c}} \phi_{-1,-\frac{1}{2}}^{\Sigma_{c}}-\phi_{1,-\frac{1}{2}}^{\Sigma_{c}} \phi_{-1, \frac{1}{2}}^{\Sigma_{c}}-\phi_{0, \frac{1}{2}}^{\Sigma_{c}} \phi_{0,-\frac{1}{2}}^{\Sigma_{c}}\right. \\
& \left.+\phi_{0,-\frac{1}{2}}^{\Sigma_{c}} \phi_{0, \frac{1}{2}}^{\Sigma_{c}}+\phi_{-1, \frac{1}{2}}^{\Sigma_{c}} \phi_{1,-\frac{1}{2}}^{\Sigma_{c}}-\phi_{-1,-\frac{1}{2}}^{\Sigma_{c}} \phi_{1, \frac{1}{2}}^{\Sigma_{c}}\right], \\
& \left|N \Xi_{c c}\right\rangle=\frac{1}{2}\left[\phi_{\frac{1}{2}, \frac{1}{2}}^{N} \phi_{-\frac{1}{2},-\frac{1}{2}}^{\Xi_{c c}}-\phi_{\frac{1}{2},-\frac{1}{2}}^{N} \phi_{-\frac{1}{2}, \frac{1}{2}}^{\Xi_{c c}}\right. \\
& \left.-\phi_{-\frac{1}{2}, \frac{1}{2}}^{N} \phi_{\frac{1}{2},-\frac{1}{2}}^{\Xi_{c c}}+\phi_{-\frac{1}{2},-\frac{1}{2}}^{N} \phi_{\frac{1}{2}, \frac{1}{2}}^{\Xi_{c c}}\right] \text {, } \\
& \left|\Lambda_{c} \Lambda_{c}\right\rangle=\sqrt{\frac{1}{2}}\left[\phi_{0, \frac{1}{2}}^{\Lambda_{c}} \phi_{0,-\frac{1}{2}}^{\Lambda_{c}}-\phi_{0,-\frac{1}{2}}^{\Lambda_{c}} \phi_{0, \frac{1}{2}}^{\Lambda_{c}}\right] \text {, } \\
& \left|\Sigma_{c}^{*} \Sigma_{c}^{*}\right\rangle=\sqrt{\frac{1}{12}}\left[\phi_{1, \frac{3}{2}}^{\Sigma_{c}^{*}} \phi_{-1,-\frac{3}{2}}^{\Sigma_{c}^{*}}-\phi_{1, \frac{1}{2}}^{\Sigma_{c}^{*}} \phi_{-1,-\frac{1}{2}}^{\Sigma_{c}^{*}}+\phi_{1,-\frac{1}{2}}^{\Sigma_{c}^{*}} \phi_{-1, \frac{1}{2}}^{\Sigma_{c}^{*}}\right. \\
& -\phi_{1,-\frac{3}{2}}^{\Sigma_{c}^{*}} \phi_{-1, \frac{3}{2}}^{\Sigma_{c}^{*}}-\phi_{0, \frac{3}{2}}^{\Sigma_{c}^{*}} \phi_{0,-\frac{3}{2}}^{\Sigma_{c}^{*}}+\phi_{0, \frac{1}{2}}^{\Sigma_{c}^{*}} \phi_{0,-\frac{1}{2}}^{\Sigma_{c}^{*}} \\
& -\phi_{0,-\frac{1}{2}}^{\Sigma_{c}^{*}} \phi_{0, \frac{1}{2}}^{\Sigma_{c}^{*}}+\phi_{0,-\frac{3}{2}}^{\Sigma_{c}^{*}} \phi_{0, \frac{3}{2}}^{\Sigma_{c}^{*}}+\phi_{-1, \frac{3}{2}}^{\Sigma_{c}^{*}} \phi_{1,-\frac{3}{2}}^{\Sigma_{c}^{*}} \\
& \left.-\phi_{-1, \frac{1}{2}}^{\Sigma_{c}^{*}} \phi_{1,-\frac{1}{2}}^{\Sigma_{c}^{*}}+\phi_{-1,-\frac{1}{2}}^{\Sigma_{c}^{*}} \phi_{1, \frac{1}{2}}^{\Sigma_{c}^{*}}-\phi_{-1,-\frac{3}{2}}^{\Sigma_{c}^{*}} \phi_{1, \frac{3}{2}}^{\Sigma_{c}^{*}}\right]
\end{aligned}
$$

where the expression of $\phi_{I_{z}, s_{z}}^{B}$ is shown as follows:

$$
\begin{aligned}
\phi_{1, \frac{1}{2}}^{\Sigma_{c}} & =\sqrt{\frac{1}{2}}\left(\chi_{1,1}^{f 1} \chi_{\frac{1}{2}, \frac{1}{2}}^{\sigma 1}+\chi_{1,1}^{f 2} \chi_{\frac{1}{2}, \frac{1}{2}}^{\sigma 2}\right) \chi^{c}, \\
\phi_{-1,-\frac{1}{2}}^{\Sigma_{c}} & =\sqrt{\frac{1}{2}}\left(\chi_{1,-1}^{f 1} \chi_{\frac{1}{2},-\frac{1}{2}}^{\sigma 1}+\chi_{1,-1}^{f 2} \chi_{\frac{1}{2},-\frac{1}{2}}^{\sigma 2}\right) \chi^{c}, \\
\phi_{1,-\frac{1}{2}}^{\Sigma_{c}} & =\sqrt{\frac{1}{2}}\left(\chi_{1,1}^{f 1} \chi_{\frac{1}{2},-\frac{1}{2}}^{\sigma 1}+\chi_{1,1}^{f 2} \chi_{\frac{1}{2},-\frac{1}{2}}^{\sigma 2}\right) \chi^{c}, \\
\phi_{-1, \frac{1}{2}}^{\Sigma_{c}} & =\sqrt{\frac{1}{2}}\left(\chi_{1,-1}^{f 1} \chi_{\frac{1}{2}, \frac{1}{2}}^{\sigma 1}+\chi_{1,-1}^{f 2} \chi_{\frac{1}{2}, \frac{1}{2}}^{\sigma 2}\right) \chi^{c}, \\
\phi_{0, \frac{1}{2}}^{\Sigma_{c}} & =\sqrt{\frac{1}{2}}\left(\chi_{1,0}^{f 1} \chi_{\frac{1}{2}, \frac{1}{2}}^{\sigma 1}+\chi_{1,0}^{f 2} \chi_{\frac{1}{2}, \frac{1}{2}}^{\sigma 2}\right) \chi^{c},
\end{aligned}
$$

$$
\begin{aligned}
& \phi_{0,-\frac{1}{2}}^{\Sigma_{c}}=\sqrt{\frac{1}{2}}\left(\chi_{1,0}^{f 1} \chi_{\frac{1}{2},-\frac{1}{2}}^{\sigma 1}+\chi_{1,0}^{f 2} \chi_{\frac{1}{2},-\frac{1}{2}}^{\sigma 2}\right) \chi^{c}, \\
& \phi_{\frac{1}{2}, \frac{1}{2}}^{N}=\sqrt{\frac{1}{2}}\left(\chi_{\frac{1}{2}, \frac{1}{2}}^{f 1} \chi_{\frac{1}{2}, \frac{1}{2}}^{\sigma 1}+\chi_{\frac{1}{2}, \frac{1}{2}}^{f 2} \chi_{\frac{1}{2}, \frac{1}{2}}^{\sigma 2}\right) \chi^{c}, \\
& \phi_{\frac{1}{2},-\frac{1}{2}}^{N}=\sqrt{\frac{1}{2}}\left(\chi_{\frac{1}{2}, \frac{1}{2}}^{f 1} \chi_{\frac{1}{2},-\frac{1}{2}}^{\sigma 1}+\chi_{\frac{1}{2}, \frac{1}{2}}^{f 2} \chi_{\frac{1}{2},-\frac{1}{2}}^{\sigma 2}\right) \chi^{c}, \\
& \phi_{-\frac{1}{2}, \frac{1}{2}}^{N}=\sqrt{\frac{1}{2}}\left(\chi_{\frac{1}{2},-\frac{1}{2}}^{f 1} \chi_{\frac{1}{2}, \frac{1}{2}}^{\sigma 1}+\chi_{\frac{1}{2},-\frac{1}{2}}^{f 2} \chi_{\frac{1}{2}, \frac{1}{2}}^{\sigma 2}\right) \chi^{c}, \\
& \phi_{-\frac{1}{2},-\frac{1}{2}}^{N}=\sqrt{\frac{1}{2}}\left(\chi_{\frac{1}{2},-\frac{1}{2}}^{f 1} \chi_{\frac{1}{2},-\frac{1}{2}}^{\sigma 1}+\chi_{\frac{1}{2},-\frac{1}{2}}^{f 2} \chi_{\frac{1}{2},-\frac{1}{2}}^{\sigma 2}\right) \chi^{c} \text {, } \\
& \phi_{-\frac{1}{2},-\frac{1}{2}}^{\Xi_{c c}}=\sqrt{\frac{1}{2}}\left(\chi_{\frac{1}{2},-\frac{1}{2}}^{f 3} \chi_{\frac{1}{2},-\frac{1}{2}}^{\sigma 1}+\chi_{\frac{1}{2},-\frac{1}{2}}^{f 4} \chi_{\frac{1}{2},-\frac{1}{2}}^{\sigma 2}\right) \chi^{c}, \\
& \phi_{-\frac{1}{2}, \frac{1}{2}}^{\Xi_{c c}}=\sqrt{\frac{1}{2}}\left(\chi_{\frac{1}{2},-\frac{1}{2}}^{f 3} \chi_{\frac{1}{2}, \frac{1}{2}}^{\sigma 1}+\chi_{\frac{1}{2},-\frac{1}{2}}^{f 4} \chi_{\frac{1}{2}, \frac{1}{2}}^{\sigma 2}\right) \chi^{c}, \\
& \phi_{\frac{1}{2},-\frac{1}{2}}^{\Xi_{c c}}=\sqrt{\frac{1}{2}}\left(\chi_{\frac{1}{2}, \frac{1}{2}}^{f 3} \chi_{\frac{1}{2},-\frac{1}{2}}^{\sigma 1}+\chi_{\frac{1}{2}, \frac{1}{2}}^{f 4} \chi_{\frac{1}{2},-\frac{1}{2}}^{\sigma 2}\right) \chi^{c}, \\
& \phi_{\frac{1}{2}, \frac{1}{2}}^{\Xi_{c c}}=\sqrt{\frac{1}{2}}\left(\chi_{\frac{1}{2}, \frac{1}{2}}^{f 3} \chi_{\frac{1}{2}, \frac{1}{2}}^{\sigma 1}+\chi_{\frac{1}{2}, \frac{1}{2}}^{f 4} \chi_{\frac{1}{2}, \frac{1}{2}}^{\sigma 2}\right) \chi^{c}, \\
& \phi_{0, \frac{1}{2}}^{\Lambda_{c}}=\sqrt{\frac{1}{2}}\left(\chi_{0,0}^{f 1} \chi_{\frac{1}{2}, \frac{1}{2}}^{\sigma 1}+\chi_{0,0}^{f 2} \chi_{\frac{1}{2}, \frac{1}{2}}^{\sigma 2}\right) \chi^{c} \text {, } \\
& \phi_{0,-\frac{1}{2}}^{\Lambda_{c}}=\sqrt{\frac{1}{2}}\left(\chi_{0,0}^{f 1} \chi_{\frac{1}{2},-\frac{1}{2}}^{\sigma 1}+\chi_{0,0}^{f 2} \chi_{\frac{1}{2},-\frac{1}{2}}^{\sigma 2}\right) \chi^{c}, \\
& \phi_{1, \frac{3}{2}}^{\Sigma_{c}^{*}}=\chi_{1,1}^{f 3} \chi_{\frac{3}{2}, \frac{3}{2}}^{\sigma} \chi^{c}, \quad \phi_{1, \frac{1}{2}}^{\Sigma_{c}^{*}}=\chi_{1,1}^{f 3} \chi_{\frac{3}{2}, \frac{1}{2}}^{\sigma} \chi^{c}, \\
& \phi_{1,-\frac{1}{2}}^{\Sigma_{c}^{*}}=\chi_{1,1}^{f 3} \chi_{\frac{3}{2},-\frac{1}{2}}^{\sigma} \chi^{c}, \quad \phi_{1,-\frac{3}{2}}^{\Sigma_{c}^{*}}=\chi_{1,1}^{f 3} \chi_{\frac{3}{2},-\frac{3}{2}}^{\sigma} \chi^{c}, \\
& \phi_{0, \frac{3}{2}}^{\Sigma_{c}^{*}}=\chi_{1,0}^{f 3} \chi_{\frac{3}{2}, \frac{3}{2}}^{\sigma} \chi^{c}, \quad \phi_{0, \frac{1}{2}}^{\Sigma_{c}^{*}}=\chi_{1,0}^{f 3} \chi_{\frac{3}{2}, \frac{1}{2}}^{\sigma} \chi^{c}, \\
& \phi_{0,-\frac{1}{2}}^{\Sigma_{c}^{*}}=\chi_{1,0}^{f 3} \chi_{\frac{3}{2},-\frac{1}{2}}^{\sigma} \chi^{c}, \quad \phi_{0,-\frac{3}{2}}^{\Sigma_{c}^{*}}=\chi_{1,0}^{f 3} \chi_{\frac{3}{2},-\frac{3}{2}}^{\sigma} \chi^{c}, \\
& \phi_{-1, \frac{3}{2}}^{\Sigma_{c}^{*}}=\chi_{1,-1}^{f 3} \chi_{\frac{3}{2}, \frac{3}{2}}^{\sigma} \chi^{c}, \quad \phi_{-1, \frac{1}{2}}^{\Sigma_{c}^{*}}=\chi_{1,-1}^{f 3} \chi_{\frac{3}{2}, \frac{1}{2}}^{\sigma} \chi^{c}, \\
& \phi_{-1,-\frac{1}{2}}^{\Sigma_{c}^{*}}=\chi_{1,-1}^{f 3} \chi_{\frac{3}{2},-\frac{1}{2}}^{\sigma} \chi^{c}, \quad \phi_{-1,-\frac{3}{2}}^{\Sigma_{c}^{*}}=\chi_{1,-1}^{f 3} \chi_{\frac{3}{2},-\frac{3}{2}}^{\sigma} \chi^{c} .
\end{aligned}
$$

Here, we should mention that the definition of different baryon-baryon channel, like the channels $\Sigma_{c} \Sigma_{c}, N \Xi_{c c}$, and so on, just represents the coupling sequences, which are shown above. In the real baryon-baryon system, the identical quarks in different baryon cluster will exchange between two clusters due to the antisymmetrization operator. Besides, the introduction of the delocalization parameter in QDCSM also allows the quark to run between two clusters, especially when two clusters are very close, so the $\Sigma_{c} \Sigma_{c}$ channel does not simply refer to the state composed of two $\Sigma_{c}$ s. Instead, it only stands for the coupling sequence. However, when two baryon clusters are far apart, the exchange interaction between two clusters will approach zero, and the delocalization parameter will be zero too. Then the $\Sigma_{c} \Sigma_{c}$ channel in this case really represents the two $\Sigma_{c}$ baryons. For convenience, we use this kind of representation of each channel in the following discussions. 

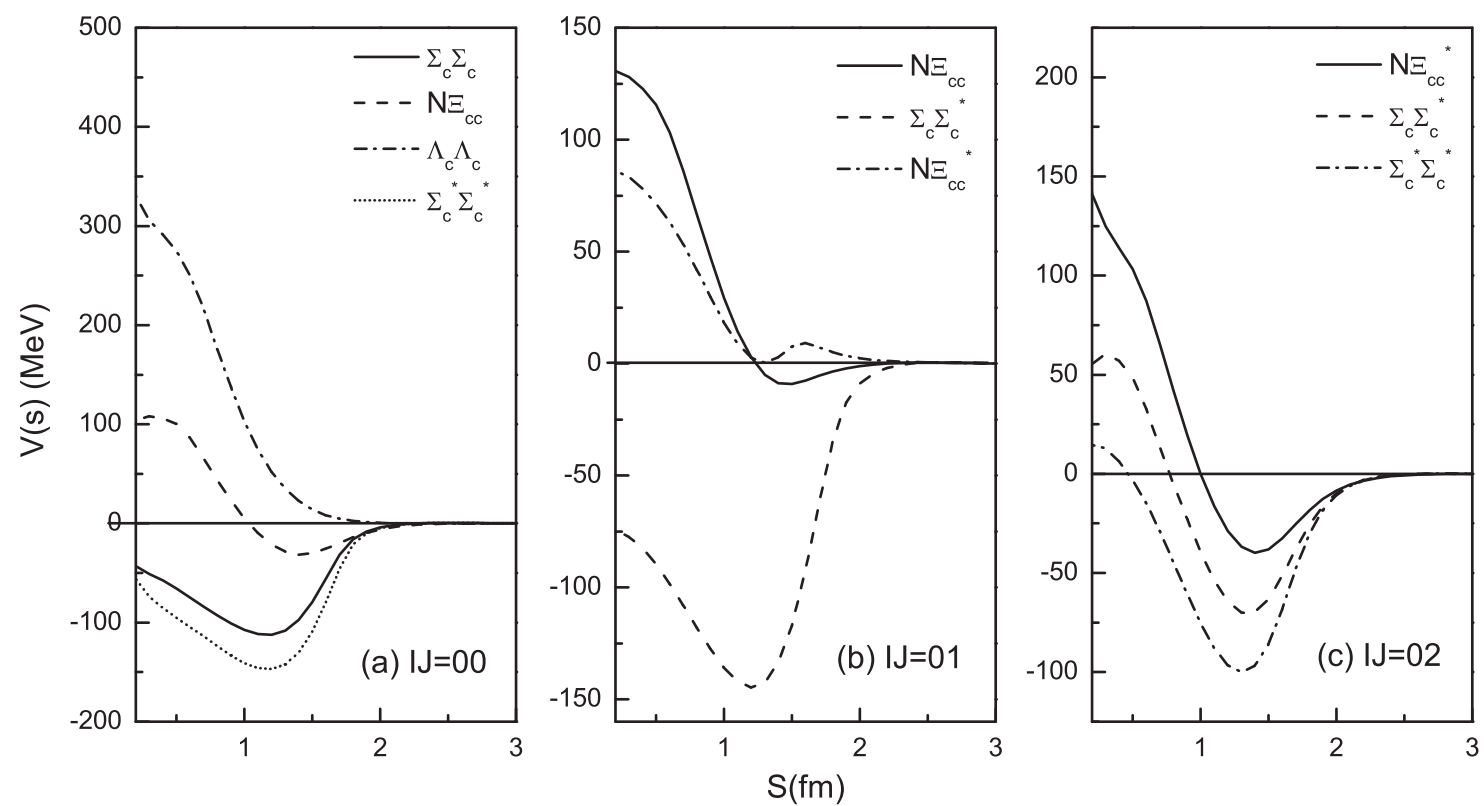

FIG. 1. The effective potentials of different channels of the doubly charm dibaryon systems with $I=0$.

\section{RESULTS AND DISCUSSION}

In this work, we perform a systematical investigation to the low-lying doubly heavy dibaryon systems with strange $S=0$, isospin $I=0,1,2$, and the angular momentum $J=0,1,2,3$. Here, we first show the results of the doubly charm dibaryon systems, and the one of the doubly bottom dibaryon systems will be shown at the end of this section.

\section{A. The effective potentials}

Since the attractive potential is necessary for forming a bound state or resonance, we first calculate the effective potential between two baryons, which is defined as $V(S)=$ $E(S)-E(\infty)$, where $E(S)$ is the diagonal matrix element of the Hamiltonian of the system in the generator coordinate. The behavior of the effective potential for the calculation with and without the heavy meson exchange is similar. To save space, we show the results including the heavy meson exchange interactions. The effective potentials of all channels with different quantum numbers are shown in Figs. 1-3, respectively.

From Figs. 1, 2, and 3, we can see that the effective potentials of several channels are purely repulsive, which are $\Lambda_{c} \Lambda_{c}$ with $I J=00, N \Xi_{c c}^{*}$ with $I J=01, N \Xi_{c c}$ and $\Lambda_{c} \Sigma_{c}$ with $I J=$ 10, $\Lambda_{c} \Sigma_{c}^{*}, N \Xi_{c c}$, and $\Lambda_{c} \Sigma_{c}$ with $I J=11, \Lambda_{c} \Sigma_{c}^{*}$ and $N \Xi_{c c}^{*}$ with $I J=12, \Delta \Xi_{c c}$ with $I J=21, \Delta \Xi_{c c}$ and $\Sigma_{c}^{*} \Sigma_{c}^{*}$ with $I J=22$, and $\Delta \Xi_{c c}^{*}$ with $I J=23$. So it is difficult for these channels to form any bound state. Conversely, the following channels have a deep effective attraction, which is larger than $-100 \mathrm{MeV}$. They are $\Sigma_{c}^{*} \Sigma_{c}^{*}$ and $\Sigma_{c} \Sigma_{c}$ with $I J=00, \Sigma_{c} \Sigma_{c}^{*}$ with $I J=01, \Sigma_{c}^{*} \Sigma_{c}^{*}$ with $I J=02, \Delta \Xi_{c c}^{*}$ with $I J=10, \Delta \Xi_{c c}^{*}$ and $\Delta \Xi_{c c}$ with $I J=11, \Delta \Xi_{c c}^{*}$ and $\Delta \Xi_{c c}$ with $I J=12$, and $\Delta \Xi_{c c}^{*}$ with $I J=13$. Such deep attraction will make these channels more likely to form bound states or resonance states. For other channels, the effective potentials are attractive too. Although the attraction is not very deep, we still need to confirm the existence of bound states or resonance states for these channels.

In addition, to study the contribution of each interaction term to the effective potentials, we also calculate the effective potentials of various terms, including the kinetic energy $\left(V_{v k}\right)$, the confinement $\left(V_{\text {con }}\right)$, the one-gluon exchange $\left(V_{\text {oge }}\right)$, the one-boson exchange $\left(V \pi\right.$ and $\left.V_{\eta}\right)$, and the heavy meson exchange $\left(V_{D}\right.$ and $\left.V_{\eta_{c}}\right)$. To save space, we take the $I J=01$ system as an example here. The contributions of various interaction terms to the effective potentials of the $I J=01$ system are shown in Fig. 4, where Figs. 4(a), 4(b), and 4(c) show the contributions to the single channel $N \Xi_{c c}, \Sigma_{c} \Sigma_{c}^{*}$, and $N \Xi_{c c}^{*}$, respectively, and Fig. 4(d) shows the contributions to the lowest state of the system after the channel coupling.

From the Fig. 4, we can see that the kinetic energy term provides an intermediate-range attraction for each channel, while the confinement interaction provides the short-range attraction. The contributions of the $\eta, D$, and $\eta_{c}$ meson exchange interactions to the effective potentials are relatively small. For the $N \Xi_{c c}$ and $N \Xi_{c c}^{*}$ channels, both the one-gluon exchange and the $\pi$ exchange terms provide the repulsive interactions, which weaken the overall attraction. Although the $\eta_{c}$ meson exchange provides attractive interaction, it is relatively small. So the interaction for the $N \Xi_{c c}$ channel is weakly attractive, and the one for the $N \Xi_{c c}^{*}$ channel is repulsive. For the $\Sigma_{c} \Sigma_{c}^{*}$ channel, the attraction from the kinetic energy term is much larger than that of the other two channels. Besides, both the one-gluon exchange and the $\pi$ exchange terms provide the attractive interactions. Although the $\eta_{c}$ meson exchange provides repulsive interaction, it is still very small. So the total interaction for the $\Sigma_{c} \Sigma_{c}^{*}$ channel is deeply attractive. After the channel-coupling calculation, the behavior of the potentials of the lowest state of the system is similar to the one of the $N \Xi_{c c}$ channel, but the attraction of the kinetic energy term becomes larger, which leads to the total attraction becomes a little deeper. 

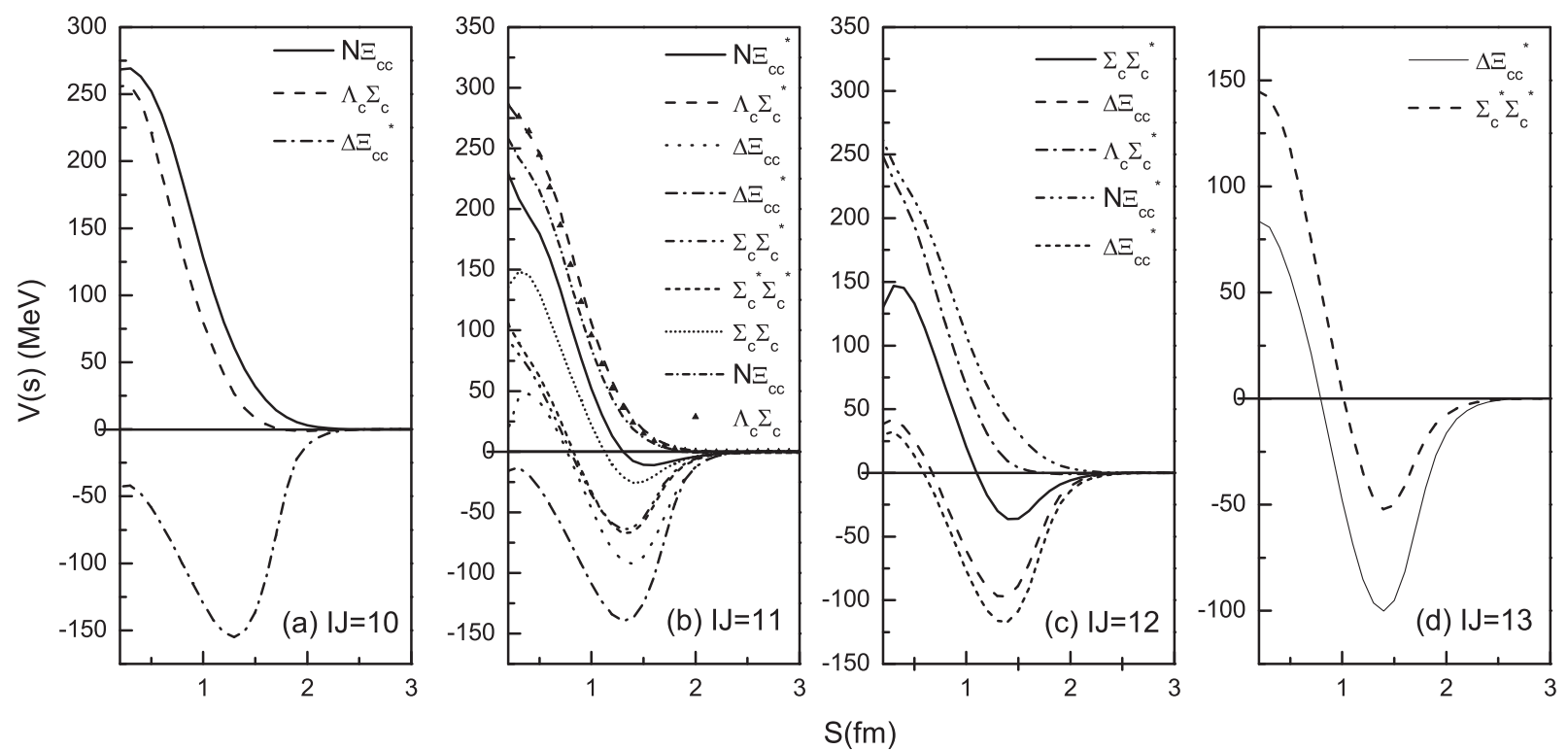

FIG. 2. The effective potentials of different channels of the doubly charm dibaryon systems with $I=1$.

From the above discussion, we can see that the kinetic energy interaction plays an important role in providing attractions, which relates to the intermediate-range attraction mechanism in QDCSM. In QDCSM, two ingredients were introduced: quark delocalization and color screening; the former is to enlarge the model variational space to take into account the mutual distortion or the internal excitations of nucleons in the course of interaction, and the latter is assuming that the quark-quark interaction dependents on quark states and aims to take into account the QCD effect, which has not been included in the two-body confinement and effective one-gluon exchange yet. In this model, the intermediate-range attraction is achieved by the quark delocalization, which is like the electron delocalization in molecules. The color screening is needed to make the quark delocalization effective.

\section{B. The bound-state calculation}

In order to see whether there is any bound state, a dynamic calculation based on the resonating group method (RGM) [51] has been performed. The energies of each channel as well as the one with channel-coupling calculation are listed in Table III. To compare the quantitative results of the calculation without and with the heavy meson interaction, the results of both cases are shown in Table III. The first column is the quantum number of the system; the second column is the corresponding state of every channel; the third column $E_{\text {th }}$ denotes the theoretical threshold of each state; the fourth column $E_{\mathrm{sc}}$ represents the energy of every single channel; the fifth column $B_{\mathrm{sc}}$ stands for the binding energy of every single channel, which is $B_{\mathrm{sc}}=E_{\mathrm{sc}}-E_{\mathrm{th}}$; the sixth column $E_{c c}$ denotes the lowest energy of the system by channel-coupling
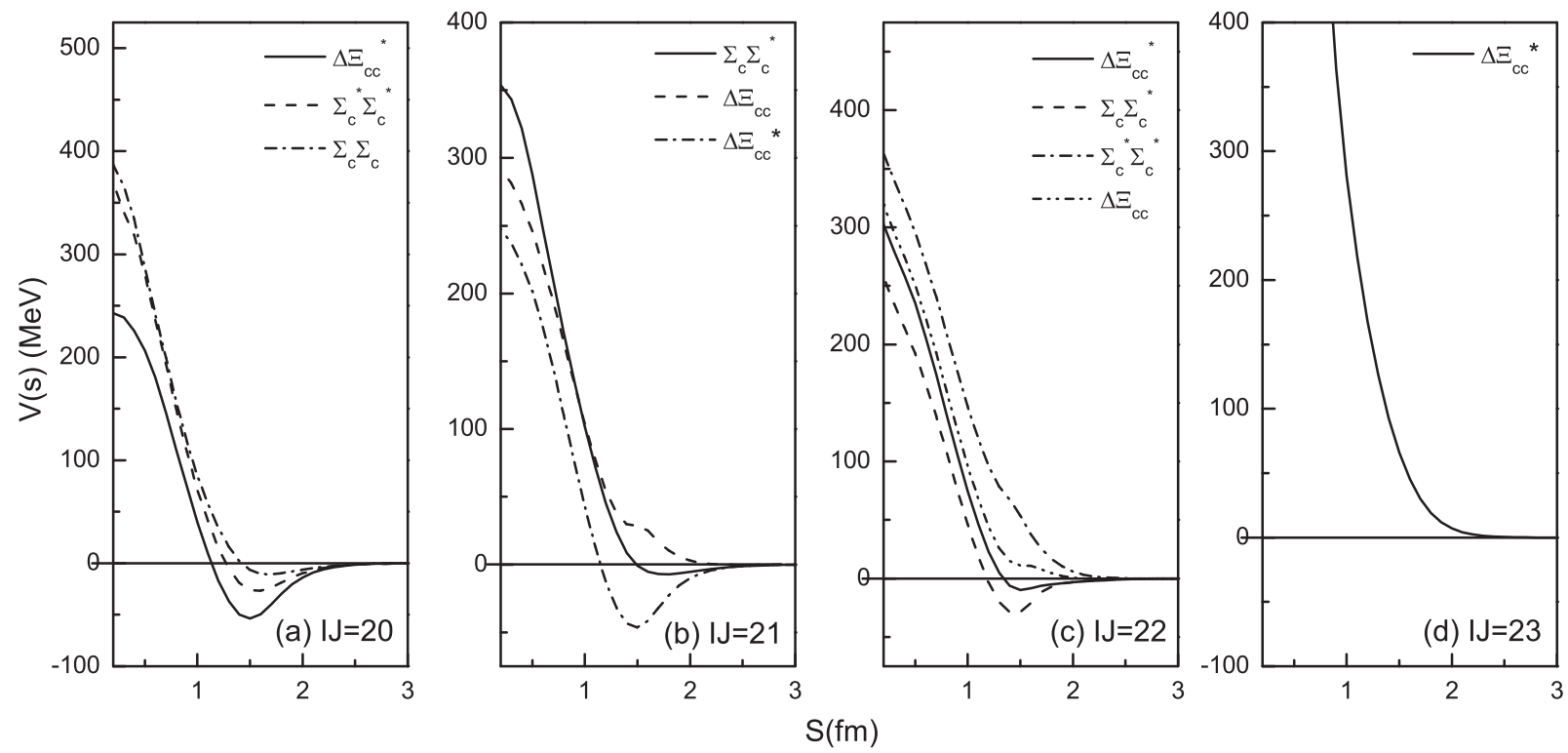

FIG. 3. The effective potentials of different channels of the doubly charm dibaryon systems with $I=2$. 

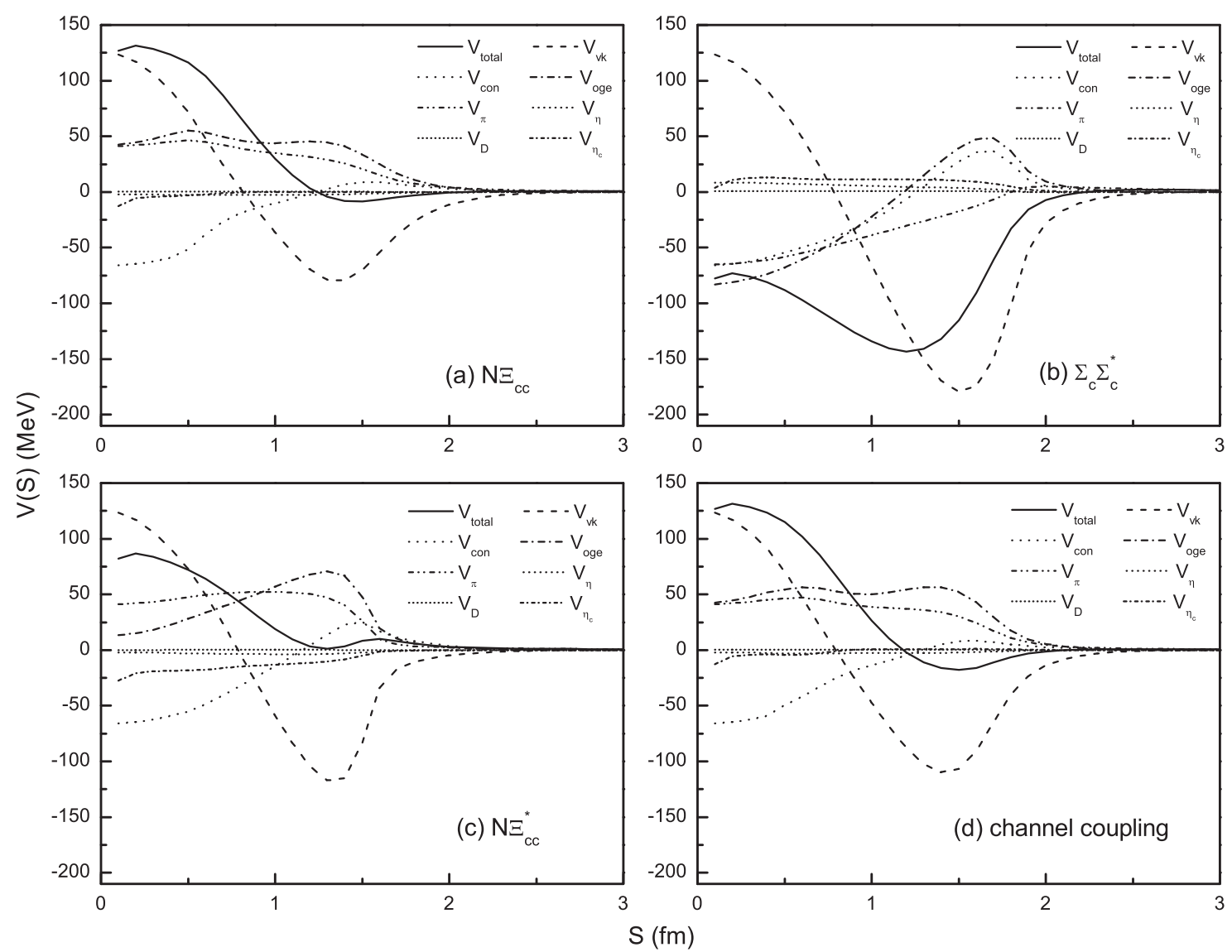

FIG. 4. The contributions to the effective potential from various terms of interactions for the $I J=01$ system.

calculation; and the last column $B_{c c}$ represents the binding energy with all channels coupling, which is $B_{c c}=E_{c c}-E_{\mathrm{th}}$. Here, we should notice that the positive value of the binding energy $B_{\mathrm{sc}}$ or $B_{c c}$ means that the state is unbound, so we label it as "ub" in Table III. In addition, the channel with the lowest threshold of each system is bolded in order to distinguish it clearly in the table.

For the system with $I J=00$, the single-channel calculation shows that both the $\Sigma_{c} \Sigma_{c}$ and $\Sigma_{c}^{*} \Sigma_{c}^{*}$ are bound states, with binding energies of $-75 /-51 \mathrm{MeV}$ and $-87 /-85 \mathrm{MeV}$, respectively, while both $N \Xi_{c c}$ and $\Lambda_{c} \Lambda_{c}$ are unbound. This is reasonable. As shown in Fig. 1(a), the interaction for the $\Sigma_{c} \Sigma_{c}$ and $\Sigma_{c}^{*} \Sigma_{c}^{*}$ channels is strong enough to form the bound state, while the attraction for the $N \Xi_{c c}$ channel is too weak to form a bound state. together. At the same time, due to the repulsive interaction of the $\Lambda_{c} \Lambda_{c}$ channel, the energy of $\Lambda_{c} \Lambda_{c}$ is above its threshold. However, the effect of the channel coupling cannot be ignored. By coupling these four channels, the lowest energy of the system is $19 / 10 \mathrm{MeV}$ lower than the threshold of $\Lambda_{c} \Lambda_{c}$, which means that the doubly charm dibaryon system with $I J=00$ is bound. This conclusion is consistent with the one on the hadron level [27]. However, the effect of the channel coupling is different between these two approaches. As we analyzed in Ref. [34], the role of the central force is much more important than the tensor force in our quark level calculation, while in the calculation on the hadron level [27], the tensor force is shown to be important and the $D$-wave channels are crucial in binding two $\Lambda_{c}$ 's. In the framework of the one-boson-exchange model, although Ref. [28] has no brief for the existence of the $\Lambda_{c} \Lambda_{c}$ state, their follow-up study supported the existence of this loosely bound state $\Lambda_{c} \Lambda_{c}$ by performing an extensive coupled channel analysis of the system [29], which also shows that the effect of the channel coupling is important for this system. However, the results of Ref. [35] pointed to the nonexistence of a charmed $H$-like dibaryon $\Lambda_{c} \Lambda_{c}$ by using a chiral constituent quark model. In their study, there is no coupling between close mass thresholds, like $\Lambda_{c} \Lambda_{c} \leftrightarrow N \Xi_{c c}$, the closest threshold being more than $300 \mathrm{MeV}$ above, so the interaction is weaker than in the strange sector. Nevertheless, the interaction in the $I J=00$ system is attractive, so this state may appear as a resonance above the $\Lambda_{c} \Lambda_{c}$ threshold [35]. Besides, the $\Sigma_{c} \Sigma_{c}$ and $\Sigma_{c}^{*} \Sigma_{c}^{*}$ channels are possible to be the resonance states by coupling to the open channels. The work of searching for the resonance states will be discussed in the next subsection.

For the system with $I J=01$, it includes three channels: $N \Xi_{c c}, \Sigma_{c} \Sigma_{c}^{*}$, and $N \Xi_{c c}^{*}$. The single-channel calculation shows that $\Sigma_{c} \Sigma_{c}^{*}$ is bound, and the binding energy is $-94 /-82 \mathrm{MeV}$ for the cases of without/with the heavy meson exchange interactions, respectively. The other two channels are unbound. After the channel-coupling calculation, the lowest energy of this system is $4733 \mathrm{MeV}$ (still higher than the threshold of the lowest channel $N \Xi_{c c}$ ) for the case of without the heavy meson exchange interactions, while it is $4720 \mathrm{MeV}$ (15 MeV lower 
TABLE III. The energy without/with the heavy meson interaction for the doubly charm dibaryon systems.

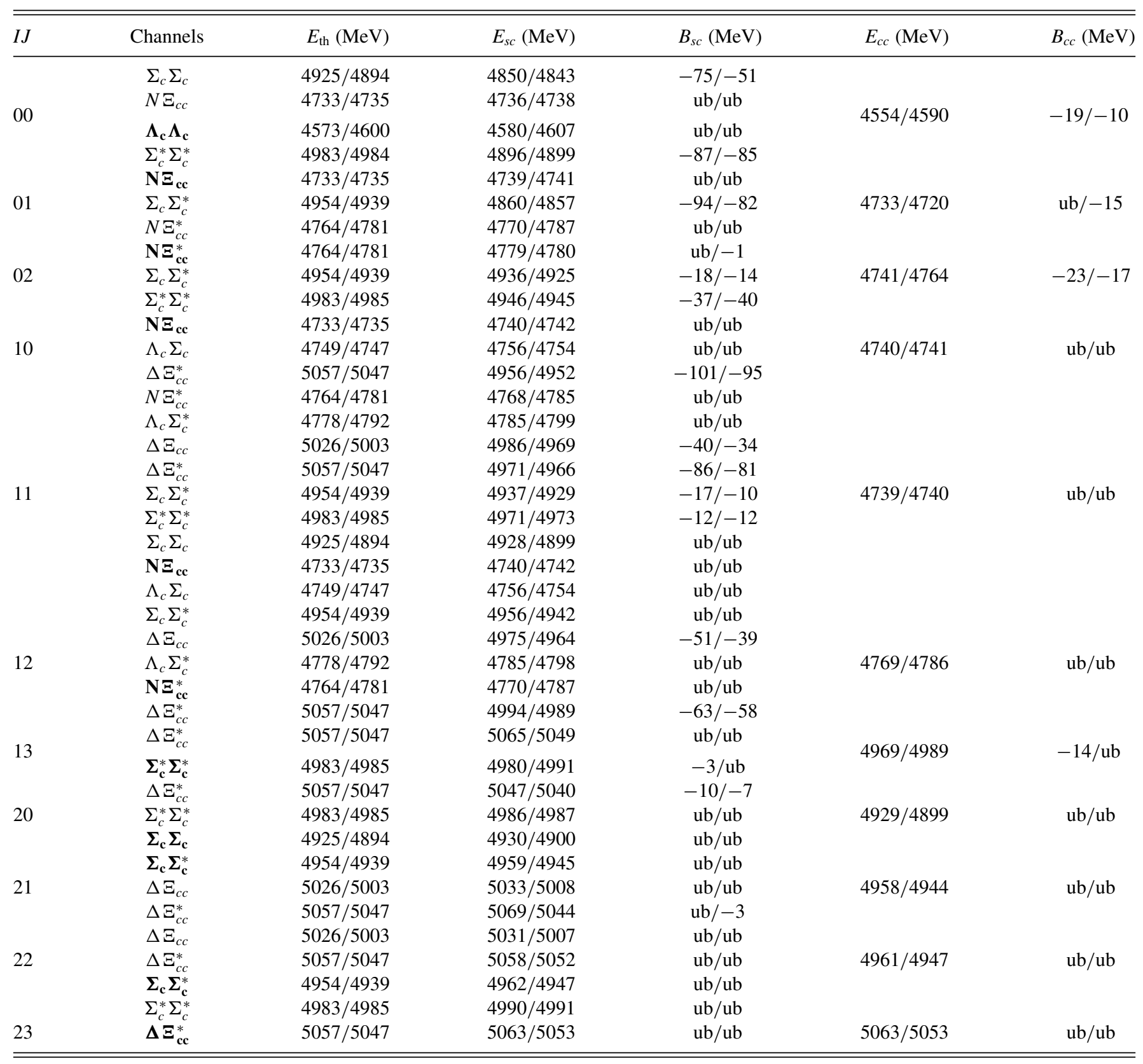

than the threshold of $N \Xi_{c c}$ ) by including the heavy meson exchange interactions. This is mainly due to the effect of the heavy meson exchange interactions. In Fig. 4(b), the heavy meson exchange provides repulsive interaction for the $\Sigma_{c} \Sigma_{c}^{*}$ channel. Although it is small, it can still reduce the binding energy of the single $\Sigma_{c} \Sigma_{c}^{*}$ channel. That is why the binding energy of the $\Sigma_{c} \Sigma_{c}^{*}$ channel becomes a little smaller after including the heavy meson exchange interaction. In contrast, the heavy meson exchange provides attractive interaction for both the $N \Xi_{c c}$ and $N \Xi_{c c}^{*}$ channels. Although it is not strong enough to form a bound state for the single channel $N \Xi_{c c}$ or $N \Xi_{c c}^{*}$, it also affects the channel-coupling calculation, and helps to form the bound state for the $I J=01$ system. So, for the doubly charmed dibaryon systems, the interaction of the heavy meson exchange can alter the binding energy because there are more heavy-light pairs in the six-quark system than in two separate baryons.

For the system with $I J=02$, it includes three channels: $N \Xi_{c c}^{*}, \Sigma_{c} \Sigma_{c}^{*}, \Sigma_{c}^{*} \Sigma_{c}^{*}$. The single-channel calculation shows that the $N \Xi_{c c}^{*}$ is unbound without the heavy meson exchange, but the energy is $1 \mathrm{MeV}$ lower than the threshold of $N \Xi_{c c}^{*}$ by considering the heavy meson exchange. Both the single channels $\Sigma_{c} \Sigma_{c}^{*}$ and $\Sigma_{c}^{*} \Sigma_{c}^{*}$ are bound with the binding energies of $-18 /-14 \mathrm{MeV}$ and $-37 /-40 \mathrm{MeV}$, respectively. After the channel-coupling calculation, the lowest energy of the system is pushed to $4741 / 4764 \mathrm{MeV}, 23 / 17 \mathrm{MeV}$ lower than the threshold of the lowest channel $N \Xi_{c c}^{*}$, which indicates that the doubly charm dibaryon system with $I J=02$ is bound.

For the system with $I J=10$, although the single channel $\Delta \Xi_{c c}^{*}$ is bound, the lowest energy of the system is higher than 
the threshold of the $N \Xi_{c c}$ channel by the channel-coupling calculation, so the system with $I J=10$ is unbound.

For the system with $I J=11$, there are nine channels as shown in Table III. The single-channel calculation shows that four states $\Delta \Xi_{c c}, \Delta \Xi_{c c}^{*}, \Sigma_{c} \Sigma_{c}^{*}$, and $\Sigma_{c}^{*} \Sigma_{c}^{*}$ are bound. However, they will become resonance states or unbound states by channel-coupling calculation, which will be discussed in the next subsection. The lowest energy of this system is $4739 / 4740 \mathrm{MeV}$ after the channel-coupling calculation, higher than the threshold of the lowest channel $N \Xi_{c c}$, which indicates that the doubly charm dibaryon system with $I J=11$ is unbound.

For the system with $I J=12$, the situation is similar to that of the $I J=10$ system. Although the single channel $\Delta \Xi_{c c}^{*}$ is bound, the lowest energy of the system is higher than the threshold of the $N \Xi_{c c}^{*}$ channel by the channel-coupling calculation. So the system with $I J=12$ is unbound.

For the system with $I J=13$, it includes two channels: $\Delta \Xi_{c c}^{*}$ and $\Sigma_{c}^{*} \Sigma_{c}^{*}$. For the calculation without the heavy meson exchange, the single channel $\Sigma_{c}^{*} \Sigma_{c}^{*}$ is bound with a binding energy of $-3 \mathrm{MeV}$. After the channel-coupling calculation, the lowest energy of the system is reduced to $4969 \mathrm{MeV}$, $14 \mathrm{MeV}$ lower than the threshold of the lower channel $\Sigma_{c}^{*} \Sigma_{c}^{*}$. However, the heavy meson exchange provides the repulsive interaction for the $\Sigma_{c}^{*} \Sigma_{c}^{*}$ channel, which leads to the unbound result for this system.

For the system with $I J=20$, the result is similar to that of the $I J=10$ system. Although the single channel $\Delta \Xi_{c c}^{*}$ is bound, there is no any bound state by the channel-coupling calculation. Besides, the results of systems with quantum numbers of $I J=21,22$, and 23 are similar. There are no bound states in any of these systems.

\section{The resonance states}

As mentioned above, some channels are bound because of the strong attractions of the system. However, these states will decay to the corresponding open channels by coupling to open channels and become resonance states. Besides, some states will become scattering state by the effect of coupling to both the open and closed channels. Another factor affects the results in some calculation is the finite coordinate space used. In quark model calculation, all the eigenenergies are discrete due to the finite space. A stabilization method [54] has been invented to pick up the genuine resonance states from these states with discrete energies. In this method, with the increase of the distance between two clusters, the continuum state will fall off toward its threshold, the energy of the bound state will remains unchanged, while a resonance state will tend to be stable. In this situation, the resonance line acts as an avoid-crossing structure and it will appear repeatedly with the increment of the distance between two clusters. Then the resonance line corresponds to the energy of the resonance state. This method has been successfully applied to the pentaquark systems $[55,56]$, the fully heavy tetraquark systems [57], and the $c \bar{c} s \bar{s}$ tetraquark systems [58]. Here, we calculate the energy eigenvalues of the doubly charm dibaryon systems by taking the value of the largest distance $\left(S_{m}\right)$ between two clusters from 4.5 to $8.5 \mathrm{fm}$ to see if there is any resonance

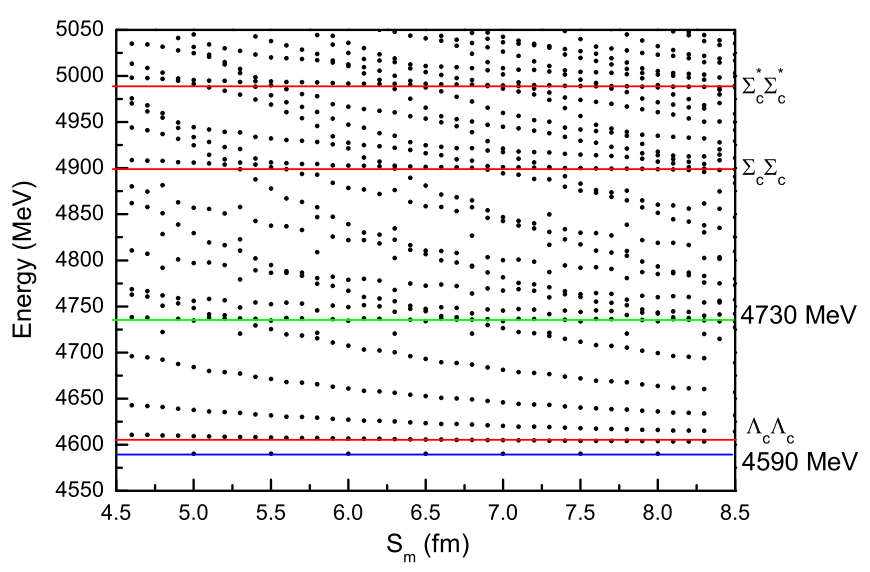

FIG. 5. The stabilization plots of the energies of the doubly charm dibaryon systems with $I J=00$.

state. To save space, we take the results of the systems with $I J=00$ and 01 as examples, which are shown in Figs. 5 and 6 , respectively. Besides, the results we show below are all include the interaction of the heavy meson exchange.

For the doubly charm dibaryon system with $I J^{P}=00$ in Fig. 5, it is clearly that the first horizontal line locates below the threshold of the lowest channel $\Lambda_{c} \Lambda_{c}$, which represents the bound state of this system. We mark it with a blue line. Then, three horizontal lines are located at the corresponding physical threshold of three channels $\Lambda_{c} \Lambda_{c}, \Sigma_{c} \Sigma_{c}$, and $\Sigma_{c}^{*} \Sigma_{c}^{*}$. We mark them with red lines. Besides, there is a line around $4730 \mathrm{MeV}$, which is stable with the variation of the distance between two clusters, so it is on behalf of a resonance state. We mark it with a green line. All these results show that after the channel coupling, neither the previously bound single channel $\Sigma_{c} \Sigma_{c}$ nor $\Sigma_{c}^{*} \Sigma_{c}^{*}$ is a resonance state, because the effect of the channel coupling raise the energies of these two channel above their thresholds. On the contrary, the effect of the channel coupling push the energy of the $N \Xi_{c c}$ channel below its threshold. So the resonance state with energy of $4730 \mathrm{MeV}$ is obtained.

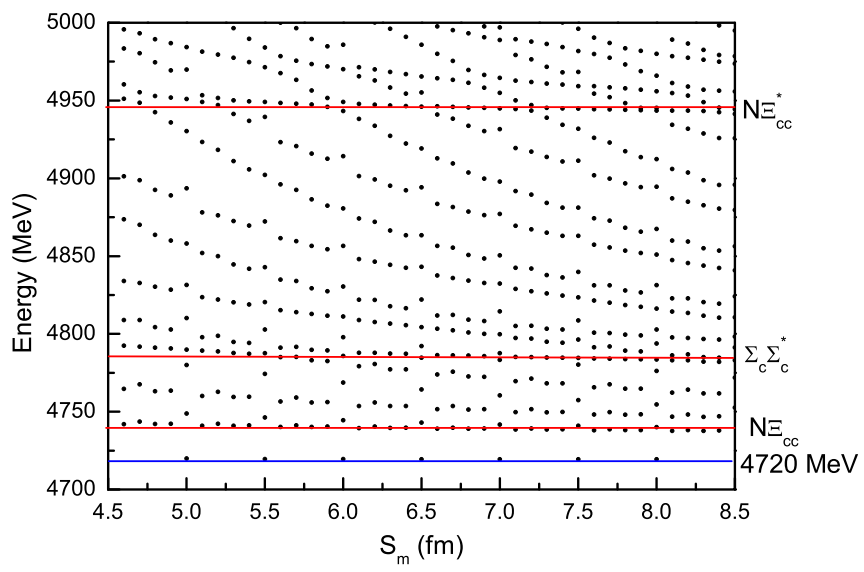

FIG. 6. The stabilization plots of the energies of the doubly charm dibaryon systems with $I J=01$. 
TABLE IV. The energy of the doubly bottom dibaryon systems.

\begin{tabular}{|c|c|c|c|c|c|c|}
\hline \multirow{5}{*}{00} & $\Sigma_{b} \Sigma_{b}$ & 11618 & 11539 & -79 & \multirow{4}{*}{11219} & \multirow{5}{*}{-20} \\
\hline & $N \Xi_{b b}$ & 11424 & 11428 & $\mathrm{ub}$ & & \\
\hline & $\Lambda_{b} \Lambda_{b}$ & 11239 & 11246 & $\mathrm{ub}$ & & \\
\hline & $\Sigma_{b}^{*} \Sigma_{b}^{*}$ & 11636 & 11553 & -83 & & \\
\hline & $\mathbf{N} \Xi_{\mathbf{b b}}$ & 11424 & 11430 & $\mathrm{ub}$ & 11428 & \\
\hline 01 & $\mathbf{N} \mathbf{\Xi}_{\mathbf{b b}}^{*}$ & 11433 & 11436 & $\mathrm{ub}$ & \multirow{4}{*}{11416} & $\mathrm{ub}$ \\
\hline \multirow[t]{3}{*}{02} & $\Sigma_{b} \Sigma_{b}^{*}$ & 11627 & 11637 & $\mathrm{ub}$ & & \multirow[t]{3}{*}{-17} \\
\hline & $\Sigma_{b}^{*} \Sigma_{b}^{*}$ & 11636 & 11646 & $\mathrm{ub}$ & & \\
\hline & $\mathbf{N} \Xi_{b b}$ & 11424 & 11431 & $\mathrm{ub}$ & & \\
\hline \multirow[t]{3}{*}{10} & $\Lambda_{b} \Sigma_{b}$ & 11428 & 11435 & $\mathrm{ub}$ & \multirow[t]{2}{*}{11430} & \multirow[t]{2}{*}{$\mathrm{ub}$} \\
\hline & $\Delta \Xi_{b b}^{*}$ & 11727 & 11635 & -92 & & \\
\hline & $\Delta \boldsymbol{\Xi}_{b b}^{*}$ & 11727 & 11648 & -79 & \multirow{6}{*}{11429} & \multirow{6}{*}{$\mathrm{ub}$} \\
\hline \multirow[t]{7}{*}{11} & $\Sigma_{b} \Sigma_{b}^{*}$ & 11627 & 11614 & -13 & & \\
\hline & $\Sigma_{b}^{*} \Sigma_{b}^{*}$ & 11636 & 11631 & -5 & & \\
\hline & $\Sigma_{b} \Sigma_{b}$ & 11618 & 11621 & $\mathrm{ub}$ & & \\
\hline & $\mathbf{N} \Xi_{\mathbf{b b}}$ & 11424 & 11431 & $\mathrm{ub}$ & & \\
\hline & $\Lambda_{b} \Sigma_{b}$ & 11428 & 11435 & $\mathrm{ub}$ & & \\
\hline & $\Sigma_{b} \Sigma_{b}^{*}$ & 11627 & 11630 & $\mathrm{ub}$ & \multirow{5}{*}{11439} & \multirow{5}{*}{$\mathrm{ub}$} \\
\hline & $\Delta \Xi_{b b}$ & 11717 & 11667 & -50 & & \\
\hline \multirow[t]{3}{*}{12} & $\Lambda_{b} \Sigma_{b}^{*}$ & 11438 & 11444 & $\mathrm{ub}$ & & \\
\hline & $\mathbf{N} \mathbf{\Xi}_{\mathbf{b b}}^{*}$ & 11433 & 11441 & $\mathrm{ub}$ & & \\
\hline & $\Delta \boldsymbol{\Xi}_{b b}^{*}$ & 11727 & 11671 & -56 & & \\
\hline 13 & $\Delta \Xi_{b b}^{*}$ & 11727 & 11689 & -38 & 11633 & -3 \\
\hline 21 & $\Delta \Xi_{b b}$ & 11717 & 11722 & $\mathrm{ub}$ & & \\
\hline 22 & $\Delta \Xi_{b b}^{*}$ & 11727 & 11731 & $\mathrm{ub}$ & 11633 & ub \\
\hline & $\Sigma_{\mathbf{b}} \Sigma_{\mathbf{b}}^{*}$ & 11627 & 11634 & $\mathrm{ub}$ & 11053 & \\
\hline & $\Sigma_{b}^{*} \Sigma_{b}^{*}$ & 11636 & 11643 & $\mathrm{ub}$ & & \\
\hline 23 & $\Delta \boldsymbol{\Xi}_{\mathbf{b b}}^{*}$ & 11727 & 11734 & $\mathrm{ub}$ & 11734 & $\mathrm{ub}$ \\
\hline
\end{tabular}

For the doubly charm dibaryon system with $I J^{P}=01$ in Fig. 6, it is obvious that the first horizontal line locates below the threshold of the lowest channel $N \Xi_{c c}$, which stands for the bound state of this system. Besides, three horizontal lines locate at the corresponding physical threshold of three channels $N \Xi_{c c}, \Sigma_{c} \Sigma_{c}^{*}$, and $N \Xi_{c c}^{*}$. No resonance state is found here, which means that the previously bound single channel $\Sigma_{c} \Sigma_{c}^{*}$ becomes the scattering state by the effect of the channel coupling. For the systems with other quantum numbers, some single channels that were previously bound become scattering states by the effect of the channel coupling. We finally obtain another two resonance states, which are the $I J^{P}=11 N \Xi_{c c}^{*}$ channel with energy of $4775 \mathrm{MeV}$, and the $I J^{P}=12 \Sigma_{c} \Sigma_{c}^{*}$ channel with energy of $4935 \mathrm{MeV}$.

\section{The doubly bottom dibaryon systems}

Because of the heavy flavor symmetry, we also extend the study to the doubly bottom dibaryon systems. Since the $D$, $D_{s}$, and $\eta_{c}$ mesons exchange do not work in the doubly bottom dibaryon systems, we do not include these heavy meson exchanges here. All the results are listed in Table IV, which are similar to those of the doubly charm dibaryon systems. By the channel-coupling calculation, three bound systems are obtained, with the quantum numbers of $I J=00, I J=02$, and $I J=13$, and the binding energies of $-20,-17$, and $-3 \mathrm{MeV}$, respectively. Besides, three resonance states are obtained, which are $N \Xi_{b b}$ with $I J=00, N \Xi_{b b}^{*}$ with $I J=11$, and $\Sigma_{b} \Sigma_{b}^{*}$ with $I J=12$, and the resonance masses are 11411 , 11432 , and $11626 \mathrm{MeV}$, respectively. 


\section{SUMMARY}

The low-lying doubly heavy dibaryon systems with strange $S=0$, isospin $I=0,1,2$, and the angular momentum $J=0$, $1,2,3$ are systemically investigated by using the RGM in the framework of QDCSM. Our goal is to search for any bound state or resonance state of the doubly heavy dibaryon systems. The effective potential of every channel is calculated to study the interaction of the doubly heavy dibaryon systems. Both single-channel and channel-coupling calculations are performed to obtain the energy of all the systems. Meanwhile, a stabilization calculation is carried out to search for any resonance state. Besides, in order to incorporate the charm quark and study the effects of the $D, D_{s}$, and $\eta_{c}$ meson exchange interactions, we extend the model from $S U(3)$ to $S U(4)$, and add the interaction of these heavy meson interactions. We find that for the doubly charmed dibaryon systems, the interaction of the heavy meson exchange can alter the binding energy because there are more heavy-light pairs in the six-quark system than in two separate baryons.

The numerical results show that for the doubly charm dibaryon systems, there are three bound systems, the quantum numbers of which are $I J=00, I J=01$, and $I J=02$, and the energies are 4590, 4720, and $4764 \mathrm{MeV}$, respectively. Besides, three resonance states $N \Xi_{c c}, N \Xi_{c c}^{*}$ and $\Sigma_{c} \Sigma_{c}^{*}$ are obtained with the quantum numbers of $I J=00, I J=11$, and $I J=12$, and the resonance masses of 4730, 4775, and $4935 \mathrm{MeV}$, respectively. For the doubly bottom dibaryon systems, the quantum numbers of three bound systems are $I J=00, I J=$ 02 , and $I J=13$, and the energies are 11219,11416 , and
$11633 \mathrm{MeV}$, respectively. Additionally, three resonance states are $N \Xi_{b b}$ with $I J=00, N \Xi_{b b}^{*}$ with $I J=11$ and $\Sigma_{b} \Sigma_{b}^{*}$ with $I J=12$, and the resonance mass are $11411 \mathrm{MeV}, 11432 \mathrm{MeV}$, $11626 \mathrm{MeV}$, respectively. All these heavy dibaryons are worth looking for in experiments, although it will be a challenging subject.

We also find that the effect of the channel coupling is remarkable in the study of multiquark systems. In this work, some single states are not bound at first, but they become bound by the channel-coupling calculation. Meanwhile, some states, which are bound in the single-channel calculation, appear as unbound states or resonance states after the channelcoupling calculation. The main reason is that the doubly heavy dibaryon systems we investigate here is all in $S$ wave, and the channel coupling between all channels is through the central force, the role of which has been verified to be much more important than the tensor force in our quark level calculation [34]. Therefore, to explore the multiquark states, the effect of channel coupling cannot be neglected. Besides, we search for the resonance states only by changing the size of the orbital space. The study of the scattering process of the corresponding open channels is needed to confirm the existence of resonance states, which is our future work.

\section{ACKNOWLEDGMENTS}

This work is supported partly by the National Natural Science Foundation of China under Contracts No. 11675080, No. 11775118, and No. 11535005.
[1] M. Bashkanov, C. Bargholtz, M. Berlowski, D. Bogoslawsky, H. Calen, H. Clement, L. Demiroers, E. Doroshkevich, D. Duniec, C. Ekstrom, K. Fransson, L. Geren, L. Gustafsson, B. Hoistad, G. Ivanov, M. Jacewicz, E. Jiganov, T. Johansson, O. Khakimova, S. Keleta, I. Koch, F. Kren, S. Kullander, A. Kupsc, K. Lindberg, P. Marciniewski, R. Meier, B. Morosov, C. Pauly, H. Pettersson, Y. Petukhov, A. Povtorejko, A. Pricking, R. J. M. Y. Ruber, K. Schonning, W. Scobel, B. Shwartz, T. Skorodko, V. Sopov, J. Stepaniak, P. E. Tegner, P. Thorngren-Engblom, V. Tikhomirov, A. Turowiecki, G. J. Wagner, M. Wolke, J. Zabierowski, I. Zartova, and J. Zlomanczuk (CELSIUS-WASA Collaboration), Phys. Rev. Lett. 102, 052301 (2009).

[2] P. Adlarson, C. Adolph, W. Augustyniak, V. Baru, M. Bashkanov, T. Bednarski, F. S. Bergmann, M. Berowski, H. Bhatt, K.-T. Brinkmann et al. (WASA-at-COSY Collaboration), Phys. Rev. Lett. 106, 242302 (2011).

[3] P. Adlarson, W. Augustyniak, W. Bardan, M. Bashkanov, T. Bednarski, F. S. Bergmann, M. Berowski, H. Bhatt, M. Büscher, H. Calén et al. (WASA-at-COSY Collaboration), Phys. Lett. B 721, 229 (2013).

[4] P. Adlarson, W. Augustyniak, W. Bardan, M. Bashkanov, F. S. Bergmann, M. Berowski, H. Bhatt, M. Büscher, H. Calén, I. Ciepa et al. (WASA-at-COSY Collaboration), Phys. Rev. C 88, 055208 (2013).

[5] P. Adlarson, W. Augustyniak, W. Bardan, M. Bashkanov, F. S. Bergmann, M. Berowski, H. Bhatt, M. Büscher, H. Calén,
I. Ciepa et al. (WASA-at-COSY Collaboration), Phys. Rev. Lett. 112, 202301 (2014).

[6] F. J. Dyson and N. H. Xuong, Phys. Rev. Lett. 13, 815 (1964).

[7] T. Goldman, K. Maltman, G. J. Stephenson, K. E. Schmidt, and F. Wang, Phys. Rev. C 39, 1889 (1989).

[8] A. Gal and H Garcilazo, Phys. Rev. Lett. 111, 172301 (2013).

[9] M. Bashkanov, S. J. Brodsky, and H. Clement, Phys. Lett. B 727, 438 (2013).

[10] J. L. Ping, H. X. Huang, H. R. Pang, F. Wang, and C. W. Wong, Phys. Rev. C 79, 024001 (2009).

[11] H. X. Huang, J. L. Ping, and F. Wang, Phys. Rev. C 89, 034001 (2014)

[12] Y. Dong, P. Shen, F. Huang, and Z. Zhang, Phys. Rev. C 91, 064002 (2015).

[13] H. X. Chen, E. L. Cui, W. Chen, T. G. Steele, and S. L. Zhu, Phys. Rev. C 91, 025204 (2015).

[14] T. Goldman, K. Maltman, G. J. Stephenson, K. E. Schmidt, and F. Wang, Phys. Rev. Lett. 59, 627 (1987).

[15] M. Oka, Phys. Rev. D 38, 298 (1988).

[16] F. Wang, J. L. Ping, G. H. Wu, L. J. Teng, and T. Goldman, Phys. Rev. C 51, 3411 (1995).

[17] H. R. Pang, J. L. Ping, F. Wang, T. Goldman, and E. G. Zhao, Phys. Rev. C 69, 065207 (2004).

[18] M. Chen, H. X. Huang, J. L. Ping, and F. Wang, Phys. Rev. C 83, 015202 (2011).

[19] H. X. Huang, J. L. Ping, and F. Wang, Phys. Rev. C 92, 065202 (2015). 
[20] Q. B. Li and P. N. Shen, Eur. Phys. J. A 8, 417 (2000).

[21] F. Etminan, H. Nemura, S. Aoki, T. Doi, T. Hatsuda, Y. Ikeda, T. Inouef, N. Ishii, K. Murano, and K. Sasaki (HAL QCD Collaboration), Nucl. Phys. A 928, 89 (2014).

[22] T. Iritani, S. Aoki, T. Doi, F. Etminan, S. Gongyo, T. Hatsuda, Y. Ikeda, T. Inoue, N. Ishii, T. Miyamoto, and K. Sasaki (HAL QCD Collaboration), Phys. Lett. B 792, 284 (2019).

[23] J. Adam, L. Adamczyk, J. R. Adams, J. K. Adkins, G. Agakishiev, M. M. Aggarwal, Z. Ahammed, N. N. Ajitanand, I. Alekseev, D. M. Anderson et al. (STAR Collaboration), Phys. Lett. B 790, 490 (2019).

[24] S. Acharya, D. Adamová, A. Adler, J. Adolfsson, M. M. Aggarwal, G. Aglieri Rinella, M. Agnello, N. Agrawal, Z. Ahammed, S. Ahmad et al. (ALICE Collaboration), Nature (London) 588, 232 (2020).

[25] F. Fromel, B. Julia-Diaz, and D. O. Riska, Nucl. Phys. A 750, 337 (2005).

[26] Y. R. Liu and M. Oka, Phys. Rev. D 85, 014015 (2012).

[27] W. Meguro, Y. R. Liu, and M. Oka, Phys. Lett. B 704, 547 (2011).

[28] N. Lee, Z. G. Luo, X. L. Chen, and S. L. Zhu, Phys. Rev. D 84, 014031 (2011).

[29] N. Li and S. L. Zhu, Phys. Rev. D 86, 014020 (2012).

[30] B. Julia-Diaz and D. O. Riska, Nucl. Phys. A 755, 431 (2005).

[31] L. Meng, N. Li, and S. L. Zhu, Phys. Rev. D 95, 114019 (2017).

[32] B. Yang, L. Meng, and S. L. Zhu, Eur. Phys. J. A 56, 67 (2020).

[33] H. X. Huang, J. L. Ping, and F. Wang, Phys. Rev. C 87, 034002 (2013).

[34] H. X. Huang, J. L. Ping, and F. Wang, Phys. Rev. C 89, 035201 (2014).

[35] T. F. Carames and A. Valcarce, Phys. Rev. D 92, 034015 (2015).

[36] J. Vijande, A. Valcarce, J. M. Richard, and P. Sorba, Phys. Rev. D 94, 034038 (2016).

[37] S. M. Gerasyuta and E. E. Matskevich, Int. J. Mod. Phys. E 21, 1250058 (2012).

[38] H. Garcilazo, A. Valcarce, and T. F. Carames, Phys. Rev. C 92 , 024006 (2015).
[39] H. Garcilazo and A. Valcarce, Eur. Phys. J. C 80, 720 (2020).

[40] H. Huang, J. Ping, and F. Wang, Phys. Rev. C 101, 015204 (2020).

[41] P. Junnarkar and N. Mathur, Phys. Rev. Lett. 123, 162003 (2019).

[42] J. M. Richard, A. Valcarce, and J. Vijande, Phys. Rev. Lett. 124, 212001 (2020).

[43] H. X. Huang, J. L. Ping, X. M. Zhu, and F. Wang, arXiv:2011.00513.

[44] F. Wang, G. H. Wu, L. J. Teng, and T. Goldman, Phys. Rev. Lett. 69, 2901 (1992).

[45] A. Valcarce, H. Garcilazo, F. Fernández, and P. Gonzalez, Rep. Prog. Phys. 68, 965 (2005) and references therein.

[46] J. Vijande, F. Fernandez, and A. Valcarce, J. Phys. G 31, 481 (2005).

[47] L. Y. Glozman and D. O. Riska, Nucl. Phys. A 603, 326 (1996).

[48] F. Stancu, Eur. Phys. J. C 79, 957 (2019).

[49] C. Patrignani, K. Agashe, G. Aielli, C. Amsler, M. Antonelli, D. M. Asner, H. Baer, Sw. Banerjee, R. M. Barnett, T. Basaglia et al., Particle Data Group, Chinese Phys. C 40, 100001 (2016).

[50] J. A. Wheeler, Phys. Rev. 52, 1083 (1937).

[51] M. Kamimura, Supp. Prog. Theo. Phys. 62, 236 (1977).

[52] D. L. Hill and J. A. Wheeler, Phys. Rev. 89, 1102 (1953); J. J. Griffin and J. A. Wheeler, ibid. 108, 311 (1957).

[53] M. M. Xu, M. Yu, and L. S. Liu, Phys. Rev. Lett. 100, 092301 (2008).

[54] J. Simon, J. Chem. Phys. 75, 2465 (1981).

[55] E. Hiyama, M. Kamimura, A. Hosaka, H. Toki, and M. Yahiro, Phys. Lett. B 633, 237 (2006).

[56] E. Hiyama, A. Hosaka, M. Oka, and J. M. Richard, Phys. Rev. C 98, 045208 (2018).

[57] X. Jin, Y. Xue, H. Huang, and J. Ping, Eur. Phys. J. C 80, 1083 (2020).

[58] X. J. Liu, H. X. Huang, J. L. Ping, D. Y. Chen, and X. M. Zhu, Eur. Phys. J. C 81, 950 (2021). 